\title{
SMA observations of polarized dust emission in solar-type Class 0 protostars: Magnetic field properties at envelope scales
}

\author{
Maud Galametz ${ }^{1}$, Anaëlle Maury ${ }^{1,5}$, Josep M. Girart ${ }^{2,3}$, Ramprasad Rao ${ }^{4}$, Qizhou Zhang ${ }^{5}$, Mathilde Gaudel ${ }^{1}$, \\ Valeska Valdivia $^{1}$, Eric Keto ${ }^{5}$, and Shih-Ping Lai ${ }^{6}$ \\ 1 Astrophysics department, CEA/DRF/IRFU/DAp, Université Paris Saclay, UMR AIM, 91191 Gif-sur-Yvette, France \\ e-mail: galametz@cea.fr \\ 2 Institut de Ciències de l'Espai (ICE, CSIC), Can Magrans, S/N, 08193 Cerdanyola del Vallès, Catalonia, Spain \\ 3 Institut d'Estudis Espacials de de Catalunya (IEEC), 08034 Barcelona, Catalonia, Spain \\ 4 Institute of Astronomy and Astrophysics, Academia Sinica, 645 N. Aohoku Pl, Hilo, HI 96720, USA \\ 5 Harvard-Smithsonian Center for Astrophysics, 60 Garden street, Cambridge MA 02138, USA \\ ${ }^{6}$ Institute of Astronomy and Department of Physics, National Tsing Hua University, Hsinchu 30013, Taiwan
}

Received 12 March 2018 / Accepted 15 April 2018

\begin{abstract}
Aims. Although from a theoretical point of view magnetic fields are believed to play a significant role during the early stages of star formation, especially during the main accretion phase, the magnetic field strength and topology is poorly constrained in the youngest accreting Class 0 protostars that lead to the formation of solar-type stars.

Methods. We carried out observations of the polarized dust continuum emission with the SMA interferometer at $0.87 \mathrm{~mm}$ to probe the structure of the magnetic field in a sample of 12 low-mass Class 0 envelopes in nearby clouds, including both single protostars and multiple systems. Our SMA observations probed the envelope emission at scales $\sim 600-5000$ au with a spatial resolution ranging from 600 to 1500 au depending on the source distance.

Results. We report the detection of linearly polarized dust continuum emission in all of our targets with average polarization fractions ranging from $2 \%$ to $10 \%$ in these protostellar envelopes. The polarization fraction decreases with the continuum flux density, which translates into a decrease with the $\mathrm{H}_{2}$ column density within an individual envelope. Our analysis show that the envelope-scale magnetic field is preferentially observed either aligned or perpendicular to the outflow direction. Interestingly, our results suggest for the first time a relation between the orientation of the magnetic field and the rotational energy of envelopes, with a larger occurrence of misalignment in sources in which strong rotational motions are detected at hundreds to thousands of au scales. We also show that the best agreement between the magnetic field and outflow orientation is found in sources showing no small-scale multiplicity and no large disks at $\sim 100$ au scales.
\end{abstract}

Key words. stars: formation - circumstellar matter - ISM: magnetic fields - polarization - techniques: polarimetric

\section{Introduction}

Understanding the physical processes at work during the earliest phase of star formation is key to characterize the typical outcome of the star formation process, put constraints on the efficiency of accretion and ejection mechanisms, and determine the pristine properties of planet-forming material. Class 0 objects are the youngest accreting protostars Andre et al. 1993, 2000. The bulk of their mass resides in a dense envelope that is being actively accreted onto the central protostellar embryo during a short main accretion phase $\left(t<10^{5} \mathrm{yr}\right.$; Maury et al. 2011; Evans et al. 2009). The physics at work to reconcile the order-ofmagnitude difference between the large angular momentum of prestellar cores (Goodman et al. 1993; Caselli et al. 2002) and the rotation properties of the young main sequence stars (also called the "angular momentum problem"; Bodenheimer 1995; Belloche 2013) is still not fully understood. It was proposed that the angular momentum could be strongly reduced by the fragmentation of the core in multiple systems or the formation of a protostellar disk and/or launch of a jet carrying away angular momentum. None of these solutions, however, seem to be able to reduce the angular momentum of the core's material by the required 5 to 10 orders of magnitude.
Magnetized models have shown that the outcome of the collapse phase can be significantly modified through magnetic braking (Galli et al. 2006; Li et al. 2014). The role of magnetic fields in Class 0 properties has also been investigated observationally. A few studies have compared the observations of Class 0 protostars to the outcome of models, either vanalytically (see, e.g., Frau et al. 2011) or from magneto-hydrodynamical simulations (see, e.g., Maury et al. 2010, 2018). These studies have shown that magnetized models of protostellar formation better reproduce the observed small-scale properties of the youngest accreting protostars, for instance the lack of fragmentation and paucity of large $\left(100<r_{\text {disk }}<500\right.$ au $)$ rotationally supported disks (Maury et al. 2010, 2014; Enoch et al. 2011; Segura-Cox et al. 2016) observed in Class 0 objects.

The polarization of the thermal dust continuum emission provides an indirect probe of the magnetic field topology since the long axis of nonspherical or irregular dust grains is suggested to align perpendicular to the magnetic field direction (Lazarian 2007). Recently, the Planck Space Observatory produced an all-sky map of the polarized dust emission at submillimeter wavelengths, revealing that the Galactic magnetic field shows regular patterns (Planck Collaboration Int. XXXV 2016). 
Observations at better angular resolution obtained from groundbased facilities seem to indicate that the polarization patterns are well ordered at the scale of HII regions or molecular cloud complexes (Curran \& Chrysostomou 2007; Matthews et al. 2009; Poidevin et al. 2010). Crutcher (2012) and references therein provides a review of magnetic fields in molecular clouds.

Few polarization observations have been performed to characterize the magnetic field topology at the protostellar envelope scales where the angular momentum problem is relevant. Submillimeter interferometric observations of the polarized dust continuum emission with the Submillimeter Array (SMA; Ho et al. 2004) and Combined Array for Research in Millimeter-wave Astronomy (CARMA; Bock et al. 2006) interferometers have led to the detections of polarized dust continuum emission toward a dozen low-mass Class 0 protostars (Rao et al. 2009; Girart et al. 2006; Hull et al. 2014); in particular these observations have led to the detection of luminous and/or massive objects because of the strong sensitivity limitations to detect a few percent of the dust continuum emission emitted by low-mass objects. By comparing the largescale $\left(\sim 20^{\prime \prime}\right)$ and small-scale $\left(\sim 2.5^{\prime \prime}\right)$ B fields of protostars at $1 \mathrm{~mm}$ (TADPOL survey), Hull et al. (2014) found that sources in which the large and small magnetic field orientations are consistent tend to have higher fractional polarization, which could be a sign of the regulating role of magnetic fields during the infall of the protostellar core. No systematic relation, however, seems to exist between the core magnetic field direction and the outflow orientation (Curran \& Chrysostomou 2007; Hull et al. 2013; Zhang et al. 2014). An hourglass shape of the magnetic line segments was observed in several protostellar cores (Girart et al. 1999, 2006; Lai et al. 2002; Rao et al. 2009; Stephens et al. 2013). This hourglass pattern, which is probably linked with the envelope contraction pulling the magnetic field lines toward the central potential well, could depend strongly on the alignment between the core rotation axis and the magnetic field direction (Kataoka et al. 2012). Observations with the Atacama Large Millimeter/submillimeter Array (ALMA) are now pushing the resolution and sensitivity enough to probe down to 100 au scales in close-by low-mass Class 0 protostars. In the massive $\left(M_{\mathrm{env}} \sim 20 M_{\odot}\right)$ binary protostar Serpens SMM1, these observations have revealed a chaotic magnetic field morphology affected by the outflow (Hull et al. 2017). In the solar-type Class 0 B335, they have, on the contrary, unveiled very ordered topologies and there is a clear transition from a large-scale B-field parallel to the outflow direction to a strongly pinched B in the equatorial plane (Maury et al. 2018).

Whether or not magnetic fields play a dominant role in regulating the collapse of protostellar envelopes needs to be further investigated from an observational perspective. In this paper, we analyze observations of the $0.87 \mathrm{~mm}$ polarized dust continuum for a sample of 12 Class 0 (single and multiple systems) protostars with the SMA interferometer (Ho et al. 2004) to increase the current statistics on the polarized dust continuum emission detected in protostellar envelopes on 750-2000 au scales. We provide details on the sample and data reduction steps in Sect. 2. We study the continuum fluxes and visibility profiles and describe the polarization results in Sect. 3 . We analyze the polarization fraction dependencies with density and the potential causes of depolarization in Sect. 4.1. In Sect. 4.2, we analyze the largeto small-scale B orientation and its variation with wavelength. We finally discuss the relation between the magnetic field orientation and both the rotation and fragmentation properties in the target protostellar envelopes. A summary of this work is presented in Sect. 5.

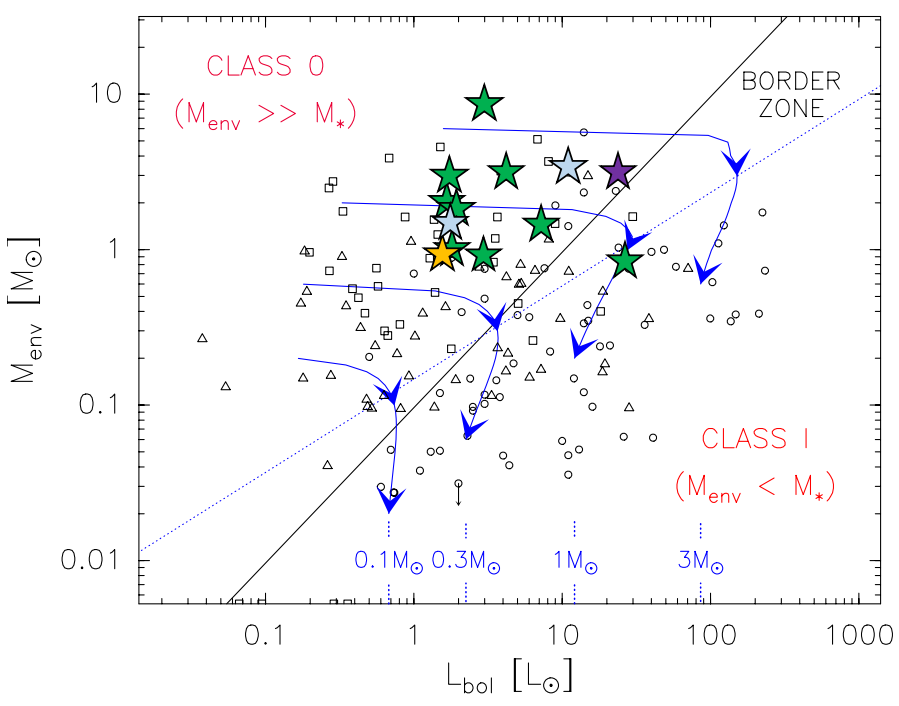

Fig. 1. Envelope mass vs. bolometric luminosity diagram from Andre et al. (2000) and Maury et al. (2011). The small black squares and triangles are Class 0 and I protostars detected in the Aquila rift, Ophiuchus, Perseus, and Orion regions (Bontemps et al. 1996; Motte \& André 2001; Andre et al. 2000; Maury et al. 2011; Sadavoy et al. 2014). The lines indicate the conceptual border zone between the Class 0 and Class I stage, with $M_{\text {env }} \propto L_{\text {bol }}$ for the dashed line and $M_{\mathrm{env}} \propto L_{\mathrm{bol}}^{0.6}$ for the dotted line. Blue lines with arrows represent protostellar evolutionary tracks, computed for final stellar masses of $0.1,0.3,1$, and $3 M_{\odot}$; the evolution proceeds from top left (objects with large envelope masses slowly accreting) to bottom (the envelope mass and the accretion luminosity are rapidly decreasing). Our sample is overlaid with the positions of protostars in our sample: B335 in yellow, IRAS16293 in purple, Perseus sources in green, and Cepheus sources in light blue. The sample only contains low-mass Class 0 protostars, except for SVS13-A. The $M_{\text {env }}$ and $L_{\text {bol }}$ are from Massi et al. (2008) for CB230, Launhardt et al. (2013) for B335, Crimier et al. (2010) for IRAS16293, Sadavoy et al. (2014), Ladjelate et al. (in prep.), and Maury et al. (in prep.) for the rest of the sources.

\section{Observations and data reduction}

\subsection{Sample description}

Our sample contains 12 protostars that include single objects as well as common-envelope multiple systems and separate envelope multiple systems (following the classification proposed by Looney et al. 2000). Figure 1 shows the location of the protostars in an envelope mass $\left(M_{\text {env }}\right)$ versus bolometric luminosity $\left(L_{\text {bol }}\right)$ diagram (Bontemps et al. 1996; Maury et al. 2011). With most of the object mass contained in the envelope, our selection is a robust sample of low-mass Class 0 protostars. Only SVS13-A can be classified as a Class I protostar. Details on each object are provided in Table 1 and in the Appendix.

\subsection{SMA $0.87 \mathrm{~mm}$ observations}

Observations of the polarized dust emission of nine low-mass protostars at $0.87 \mathrm{~mm}$ were obtained using the SMA (Projects 2013A-S034 and 2013B-S027, PI: A. Maury) in the compact and subcompact configuration. To increase our statistics, we also included SMA observations from three additional sources from Perseus (NGC 1333 IRAS4A and IRAS4B) and Ophiuchus (IRAS16293) observed in 2004 and 2006 (Projects 2004-142 and 2006-09-A026, PI: R. Rao; Project 2005-09-S061, PI: D.P. Marrone). The observations of NGC 1333 IRAS4A and IRAS16293 
Table 1. Properties of the sample.

\begin{tabular}{|c|c|c|c|c|c|}
\hline Name & Cloud & Distance $^{a}$ & Class & Multiplicity $^{b}$ & Observation Phase Reference Center \\
\hline B335 & isolated & $100 \mathrm{pc}$ & Class 0 & Single & $19 \mathrm{~h} 37 \mathrm{~m} 00.9 \mathrm{~s}+07^{\circ} 34^{\prime} 09^{\prime \prime} 6$ \\
\hline SVS13 & Perseus & $235 \mathrm{pc}$ & Class 0/I & Multiple (wide) & $03 \mathrm{~h} 29 \mathrm{~m} 03.1 \mathrm{~s}+31^{\circ} 15^{\prime} 52^{\prime \prime} 0$ \\
\hline HН797 & Perseus & $235 \mathrm{pc}$ & Class 0 & Multiple & $03 \mathrm{~h} 43 \mathrm{~m} 57.1 \mathrm{~s}+32^{\circ} 03^{\prime} 05^{\prime \prime} 6$ \\
\hline L1448C & Perseus & $235 \mathrm{pc}$ & Class 0 & Multiple (wide) & $03 \mathrm{~h} 25 \mathrm{~m} 38.9 \mathrm{~s}+30^{\circ} 45^{\prime} 14^{\prime \prime} 9$ \\
\hline L1448N & Perseus & $235 \mathrm{pc}$ & Class 0 & Multiple (close and wide) & $03 \mathrm{~h} 25 \mathrm{~m} 36.3 \mathrm{~s}+30^{\circ} 44^{\prime} 05^{\prime \prime} 4$ \\
\hline L1448-2A & Perseus & $235 \mathrm{pc}$ & Class 0 & Multiple (close and wide) & $03 \mathrm{~h} 25 \mathrm{~m} 22.4 \mathrm{~s}+30^{\circ} 45^{\prime} 12^{\prime \prime} \cdot 2$ \\
\hline IRAS03282 & Perseus & $235 \mathrm{pc}$ & Class 0 & Multiple (close) & $03 \mathrm{~h} 31 \mathrm{~m} 20.4 \mathrm{~s}+30^{\circ} 45^{\prime} 24^{\prime \prime} \cdot 7$ \\
\hline NGC 1333 IRAS4A & Perseus & $235 \mathrm{pc}$ & Class 0 & Multiple (close) & $03 \mathrm{~h} 29 \mathrm{~m} 10.5 \mathrm{~s}+31^{\circ} 13^{\prime} 31^{\prime \prime} 0$ \\
\hline NGC 1333 IRAS4B & Perseus & $235 \mathrm{pc}$ & Class 0 & Single & $03 \mathrm{~h} 29 \mathrm{~m} 12.0 \mathrm{~s}+31^{\circ} 13^{\prime} 08^{\prime \prime} 0$ \\
\hline IRAS16293 & Ophiuchus & $120 \mathrm{pc}$ & Class 0 & Multiple (wide) & $16 \mathrm{~h} 32 \mathrm{~m} 22.9 \mathrm{~s}-24^{\circ} 28^{\prime} 36^{\prime \prime} .0$ \\
\hline L1157 & Cepheus & $250 \mathrm{pc}$ & Class 0 & Single & $20 \mathrm{~h} 39 \mathrm{~m} 06.3 \mathrm{~s}+68^{\circ} 02^{\prime} 15^{\prime \prime} .8$ \\
\hline CB230 & Cepheus & $325 \mathrm{pc}$ & Class 0 & Binary (close) & $21 \mathrm{~h} 17 \mathrm{~m} 40.0 \mathrm{~s}+68^{\circ} 17^{\prime} 32^{\prime \prime} 0$ \\
\hline
\end{tabular}

Notes. ${ }^{(a)}$ Distance references are Knude \& Hog (1998), Stutz et al. (2008), Hirota et al. (2008), Hirota et al. (2011), Looney et al. (2007) and Straizys et al. (1992). ${ }^{(b)}$ Multiplicity derived from Launhardt (2004) for IRAS03282 and CB230, Rao et al. (2009) for IRAS16293, Palau et al. (2014) for HH797, Evans et al. (2015) for B335 and from the CALYPSO survey dust continuum maps at $220 \mathrm{GHz}$ (PdBI; see http://irfu . cea.fr/Projets/Calypso/, and Maury et al. 2014) for the rest of the sources.

are presented in Girart et al. (2006) and Rao et al. (2009), respectively. Marrone (2006) and Marrone \& Rao (2008) provide a detailed description of the SMA polarimeter system, but we provide a few details on the SMA and the polarization design below. The SMA has eight antennas. Each optical path is equipped with a quarter-wave plate (QWP), an optical element that adds a $90^{\circ}$ phase delay between orthogonal linear polarizations and is used to convert the linear into circular polarization. The antennas are switched between polarizations (QWP are rotated at various angles) in a coordinated temporal sequence to sample the various combinations of circular polarizations on each baseline. The 230, 345, and 400 band receivers are installed in all eight SMA antennas. Polarization can be measured in single-receiver polarization mode and in dual-receiver mode when two receivers with orthogonal linear polarizations are tuned simultaneously. In this dual-receiver mode, all correlations (the parallel-polarized RR and LL and the cross-polarized RL and LR; with R and L for right circular and left circular, respectively) are measured at the same time. Both polarization modes were used in our observations. This campaign was used to partly commission the dual-receiver full polarization mode for the SMA. A fraction of the data was lost during this period owing to issues with the correlator software. Frequent observations of various calibrators were interspersed to ensure that such issues were detected as early as possible to minimize data loss.

\subsection{Data calibration and self-calibration}

We performed the data calibration in the IDL-based software Millimeter Interferometer Reduction (MIR) and the data reduction package MIRIAD ${ }^{1}$. The calibration includes an initial flagging of high system temperatures $T_{\text {sys }}$ and other wrong visibilities, a bandpass calibration, a correction of the cross-receiver delays, a gain, and a flux calibration. The various calibrators observed for each of these steps and the list of antennae used for the observations are summarized in Table 2. The polarization

\footnotetext{
1 https://www.cfa.harvard.edu/sma/miriad/
}

calibration was performed in MIRIAD. Quasars were observed to calculate the leakage terms: the leakage amplitudes (accuracy: $\sim 0.5 \%$ ) are $<2 \%$ in the two sidebands for all antenna except for seven than can reach a few percent. Before the final imaging, we used an iterative procedure to self-calibrate the Stokes $I$ visibility data.

\subsection{Deriving the continuum and polarization maps}

The Stokes parameters describing the polarization state are defined as

$S=\left[\begin{array}{c}I \\ Q \\ U \\ V\end{array}\right]$,

with $Q$ and $U$ the linear polarization and $V$ the circular polarization. We used a robust weighting of 0.5 to transform the visibility data into a dirty map. The visibilities range from $5 \mathrm{k} \lambda$ to $30 \mathrm{k} \lambda$ in seven sources (B335, SVS13, HH797, L1448C, IRAS03282, L1157, and CB230) and $10 \mathrm{k} \lambda$ to $80 \mathrm{k} \lambda$ for the other five sources. Visibilities beyond $80 \mathrm{k} \lambda$ are available for NGC 1333 IRAS4A but not used to allow an analysis of comparable scales for all our sources. The Stokes $I$ dust continuum emission maps are shown in Fig. 2. The Stokes $Q$ and $U$ maps are shown in Fig. B.1. Their combination probes the polarized component of the dust emission. The synthesized beams and rms of the cleaned maps are provided in Table 3 . We note that because of unavoidable missing flux and the dynamic-range limitation, the rms of the Stokes $I$ maps are systematically higher than those of Stokes $Q$ and $U$. Following the self-calibration procedure, the rms of the continuum maps have decreased by $10-45 \%$.

The polarization intensity (debiased), fraction and angle are derived from the Stokes $Q$ and $U$ as follows:

$P_{i}=\sqrt{Q^{2}+U^{2}-\sigma_{Q, U}^{2}}$,

$p_{\text {frac }}=P_{i} / I$,

$P A=0.5 \times \arctan (U / Q)$, 
Table 2. Details on the observations.

\begin{tabular}{lcccccc}
\hline \hline Date & \multicolumn{1}{c}{ Mode $^{a}$} & $\begin{array}{c}\text { Flux } \\
\text { calib. }\end{array}$ & $\begin{array}{c}\text { Bandpass } \\
\text { calib. }\end{array}$ & $\begin{array}{c}\text { Gain } \\
\text { calib. }\end{array}$ & $\begin{array}{c}\text { Polarization } \\
\text { calib. }\end{array}$ & $\begin{array}{c}\text { Antenna } \\
\text { used }\end{array}$ \\
\hline Dec 05 2004 & Single Rx-Single BW & Ganymede & $3 \mathrm{c} 279$ & $3 \mathrm{c} 84$ & $3 \mathrm{c} 279$ & $1,2,3,5,6,8$ \\
Dec 06 2004 & Single Rx-Single BW & Ganymede & $3 \mathrm{c} 279$ & $3 \mathrm{c} 84$ & $3 \mathrm{c} 279$ & $1,2,3,5,6,8$ \\
April 08 2006 & Single Rx-Single BW & Callisto & $3 \mathrm{c} 273$ & $1517-243,1622-297$ & $3 \mathrm{c} 273$ & $1,2,3,5,6,7,8$ \\
Dec 23 2006 & Single Rx-Single BW & Titan & $3 \mathrm{c} 279$ & $3 \mathrm{c} 84$ & $3 \mathrm{c} 279$ & $1,2,3,4,5,6,7$ \\
Aug 26 2013 & Single Rx-Double BW & Neptune & $3 \mathrm{c} 84$ & $1927+739,0102+584$ & $3 \mathrm{c} 84$ & $2,4,5,6,7,8$ \\
Aug 31 2013 & Dual Rx-Autocorrel & Callisto & $3 \mathrm{c} 84$ & $1751+096,1927+739,0102+584,3 \mathrm{c} 84$ & $3 \mathrm{c} 84$ & $2,4,5,6,7,8$ \\
Sept 1 2013 & Dual Rx-Autocorrel & Callisto & $3 \mathrm{c} 84$ & $1927+739,0102+584$ & $3 \mathrm{c} 84$ & $2,4,5,6,7,8$ \\
Sept 2 2013 & Dual Rx-Autocorrel & Callisto & $3 \mathrm{c} 454.3$ & $1927+739,0102+584,3 \mathrm{c} 84$ & $3 \mathrm{c} 84$ & $2,4,5,6,7,8$ \\
Sept 7 2013 & Dual Rx-Full pol. & Callisto & $3 \mathrm{c} 454.3$ & $3 \mathrm{c} 84,3 \mathrm{c} 454.3$ & $3 \mathrm{c} 454.3$ & $2,4,5,6,7,8$ \\
Dec 7 2013 & Dual Rx-Full pol. & Callisto & $3 \mathrm{c} 84$ & $3 \mathrm{c} 84$ & $3 \mathrm{c} 84$ & $2,4,5,6,7,8$ \\
Feb 24 2014 & Dual Rx-Full pol. & Callisto & $3 \mathrm{c} 279$ & $3 \mathrm{c} 84,3 \mathrm{c} 279,0927+390$ & $3 \mathrm{c} 279$ & $1,2,4,5,6,7$ \\
Feb 25 2014 & Dual Rx-Full pol. & Callisto & $3 \mathrm{c} 84$ & $3 \mathrm{c} 84$ & $3 \mathrm{c} 84$ & $1,2,4,5,6,7$ \\
\hline
\end{tabular}

Notes. ${ }^{(a)} \mathrm{Rx}=$ Receiver; $\mathrm{BW}=$ bandwidth.

with $\sigma_{Q, U}$ the average rms of the $Q$ and $U$ maps. We applied a $5 \sigma$ cutoff on Stokes $I$ and $3 \sigma$ cutoff on Stokes $Q$ and $U$ to only select locations where polarized emission is robustly detected. The polarization intensity and fraction maps are provided in Fig. B.1.

\section{Results}

\section{1. $0.87 \mathrm{~mm}$ continuum fluxes}

The $0.87 \mathrm{~mm}$ dust continuum maps are presented in Fig. 2 (the Stokes I dust continuum emission is described in Appendix A). We overlay the outflow direction (references from the literature can be found in Table 6). We provide the peak intensities and integrated $0.87 \mathrm{~mm}$ flux densities in Table 4 . We also provide the associated masses calculated using the following relation from Hildebrand (1983):

$M=\frac{S_{v} \mathcal{D} d^{2}}{\kappa_{v} B_{v}(T)}$

where $\mathrm{S}_{v}$ is the flux density, $\mathcal{D}$ the dust-to-gas mass ratio assumed to be $0.01, \mathrm{~d}$ the distance to the protostar, $\kappa_{v}$ the dust opacity (tabulated in Ossenkopf \& Henning (1994), $1.85 \mathrm{~cm}^{2} \mathrm{~g}^{-1}$ at $0.87 \mathrm{~mm})$, and $\mathrm{B}_{v}(\mathrm{~T})$ the Planck function. We choose a dust temperature of $25 \mathrm{~K}$, coherent with that observed at 1000 au in IRAS 16293 by Crimier et al. (2010). A dust temperature of $50 \mathrm{~K}$ would only decrease the gas mass reported in Table 4 by a factor of $\sim 2$.

The sensitivity of SMA observations decreases outside the primary beam (i.e., 34") and the coverage of the shortest baselines is not complete. We thus expect that only part of the total envelope flux is recovered by our SMA observations, especially for the closest sources. To estimate how much of the extended flux is missing, we compare the SMA fluxes with those obtained with the SCUBA single-dish instrument. Di Francesco et al. (2008) provided a catalog of 0.87 $\mathrm{mm}$ continuum fluxes and peak intensities for a large range of objects observed with SCUBA at 450 and $850 \mu \mathrm{m}$, including our sources. The SCUBA integrated flux uncertainties are dominated by the $15 \%$ calibration uncertainties while the absolute flux uncertainty for the SMA is $<10 \%$. Results are summarized in Table 5. We find that the SMA total flux, integrated in the reconstructed cleaned maps of the sources, accounts for $9-18 \%$ of the SCUBA total fluxes. This is consistent with the results of the PROSAC low-mass protostar survey for which only 10-20\% of the SCUBA flux is recovered with the SMA (Jørgensen et al. 2007). As far as the dust continuum peak flux densities are concerned, if we rescale the SCUBA peak flux densities to that expected in the SMA beam, assuming that the intensity scales with radius (i.e., a density dependence $\rho \propto r^{-2}$ ), we find that SCUBA and SMA peak flux densities are consistent with each other. This means that most of the envelope flux is recovered at SMA beam scales. The largest discrepancies appear for B335, NGC 1333 IRAS4A, and NGC 1333 IRAS4B. For B335, the SCUBA value is twice that derived with SMA. This indicates that for this source, one of the closest of our sample, part of the SMA continuum flux might be missing even at the peak of continuum emission, and the polarization fraction could be lower than that determined from the SMA observations (Sect. 3). For NGC 1333 IRAS4A and IRAS4B, the inverse is observed: the SMA peak value is two and four times larger than the rescaled SCUBA. The two sources are the most compact envelopes of the sample (Looney et al. 2003; Santangelo et al. 2015). The difference is thus probably due to the compact protostellar cores being diluted in the large SCUBA beam and possible contamination of the SCUBA fluxes because NGC 1333 IRAS4A and B are both located within a long $\left(1^{\prime} \times 40^{\prime \prime}\right)$ filamentary cloud extending southeast-northwest (Lefloch et al. 1998).

\subsection{Analysis of the Stokes I visibilities}

Part of the extended dust continuum emission is lost during the reconstruction of the final image itself, since an interferometer can only sparsely sample Fourier components of different spatial frequencies of the incoming signal. To analyze the impact of the map reconstruction on the flux values derived, we can compare the flux densities derived from the reconstructed map with those deduced directly from the visibility amplitude curves. The Stokes $I$ amplitudes of the visibilities, averaged every 120 s, are presented in Fig. 3. The sample includes three wide binary objects resolved by our SMA observations, i.e., L1448N, SVS13, and IRAS16293. We use the visibility data to separate the binary sources. As the sources cannot be modeled using a simple Gaussian fitting, we use the MIRIAD/imsub function to produce a subimage containing L1448N-A, SVS13-A, and IRAS16293$\mathrm{B}$ from the cleaned image. We then use MIRIAD/uvmodel to 
M. Galametz et al: Polarized dust emission in solar-type Class 0 protostars
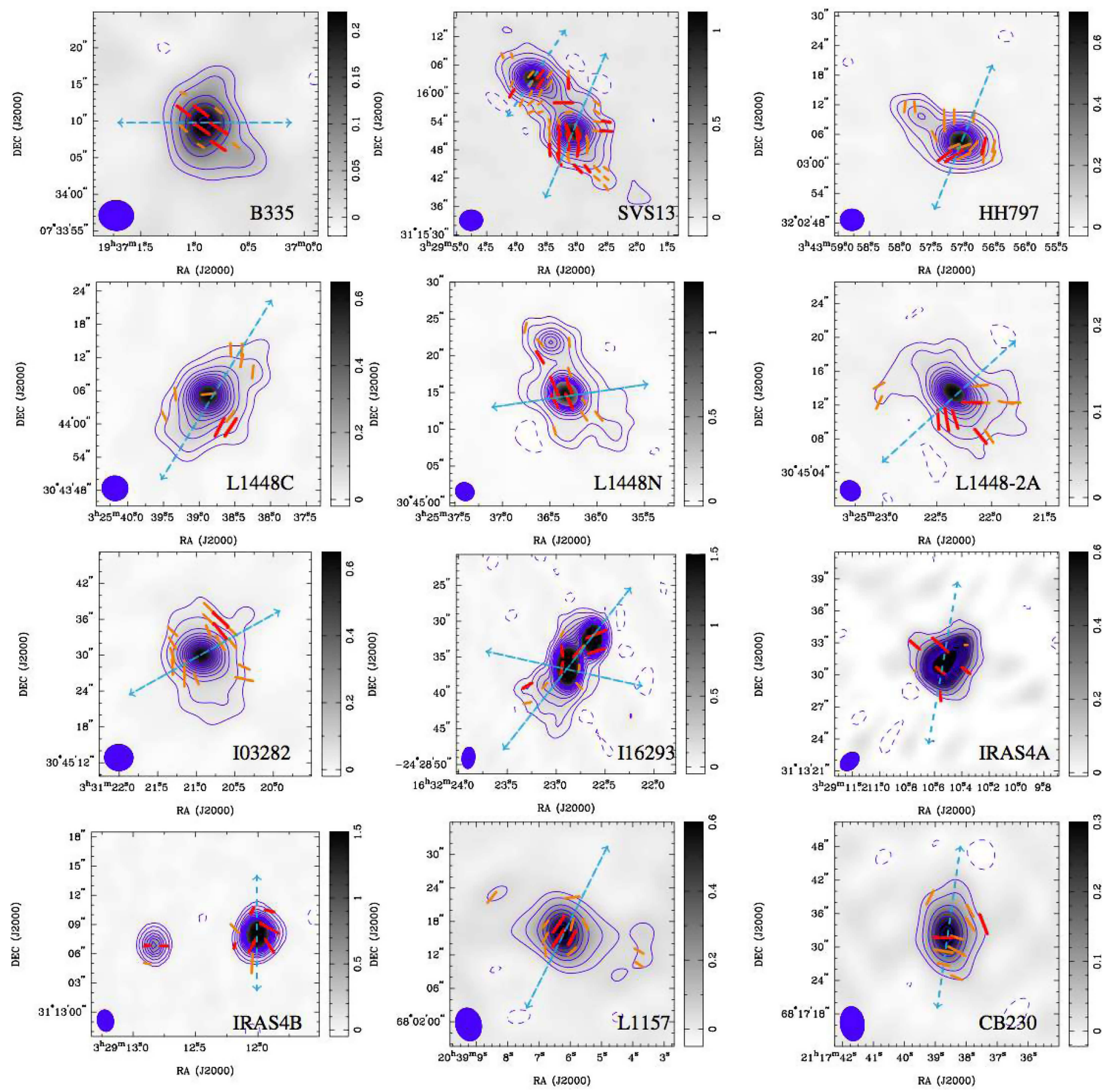

Fig. 2. SMA $850 \mu$ m Stokes $I$ continuum maps. Color scales are in mJy/beam. Contours at $[-3,5,10,20,30,40,50,60,70,80,90,100] \sigma$ appear in blue. The filled ellipses in the bottom left corner indicate the synthesized beam of the SMA maps. Their sizes are reported in Table 3. The blue arrows indicate the outflow direction. The B orientations (derived from the polarization angles assuming a $90^{\circ}$ rotation) are overlaid on the Stokes $I$ map. Red bars show $>3 \sigma$ detections. We also indicate the $>2 \sigma$ detections with orange bars but these detections are not used for this analysis

subtract these sources from the visibility data and make an image of L1448N-B, SVS13-B, and IRAS16293-A from the modified visibility datasets. We proceed the same way to map L1448N-A, SVS13-A, and IRAS16293-B, independently. The separated visibility profiles are shown in Fig. 3. The region mapped around NGC 1333 IRAS4B contained IRAS4A (outside the primary beam) and IRAS4B2 located about 10" east of IRAS4B. These two sources are also isolated and removed from the visibility data presented in Fig. 3 .
We use a Gaussian fittingv method to fit the visibilities and extrapolate these models at $0 \mathrm{k} \lambda$ to derive the integrated $0.87 \mathrm{~mm}$ continuum fluxes. The fluxes derivedv from the visibility amplitude curves are reported in Table 4 . The fluxes derived from the visibility amplitude curves are close but, as expected, systematically larger (on average 2.2 times) than those derived on the reconstructed maps. We also indicate the $0.87 \mathrm{~mm}$ continuum fluxes of L1448N-B and SVS13-B (the sources located at the phase center) in Table 4. We find that SVS13-A and B have 
Table 3. Characteristics of the SMA maps.

\begin{tabular}{|c|c|c|c|c|}
\hline \multirow[t]{2}{*}{ Name } & \multirow[t]{2}{*}{ Synthesized beam } & \multicolumn{3}{|c|}{ rms (mJy/beam) } \\
\hline & & $I^{a}$ & $Q$ & $U$ \\
\hline B335 & $44^{\prime \prime} 8 \times 44^{\prime \prime} 1\left(84^{\circ}\right)$ & 4.6 & 4.0 & 4.3 \\
\hline SVS13 & $4 ! .9 \times 4 ! .3\left(-88^{\circ}\right)$ & 7.0 & 3.0 & 3.1 \\
\hline HH797 & $4 ! .7 \times 4^{\prime \prime} 3\left(89^{\circ}\right)$ & 4.2 & 1.9 & 1.8 \\
\hline L1448C & $4 ! .7 \times 4 ! .4\left(87^{\circ}\right)$ & 4.7 & 3.6 & 3.7 \\
\hline L1448N & $2 \prime .6 \times 22^{\prime \prime} 4\left(55^{\circ}\right)$ & 5.5 & 3.3 & 3.0 \\
\hline L1448-2A & $2^{\prime \prime} .5 \times 2^{\prime \prime} .2\left(49^{\circ}\right)$ & 1.8 & 1.6 & 1.6 \\
\hline IRAS03282 & $4^{\prime \prime} 8 \times 4^{\prime \prime} 3\left(88^{\circ}\right)$ & 5.3 & 3.3 & 3.5 \\
\hline NGC 1333 IRAS4A & $2^{\prime \prime} 0 \times 11^{\prime \prime} 4\left(-46^{\circ}\right)$ & 18.7 & 7.5 & 8.0 \\
\hline NGC 1333 IRAS4B & $2, .2 \times 1^{\prime \prime} .7\left(10^{\circ}\right)$ & 9.0 & 2.2 & 2.3 \\
\hline IRAS16293 & $3 \prime \prime 0 \times 1^{\prime \prime \prime} 8\left(-6^{\circ}\right)$ & 10.4 & 5.6 & 6.0 \\
\hline L1157 & $5 \prime \prime 9 \times 4{ }^{\prime \prime} 6 \quad\left(1^{\circ}\right)$ & 8.8 & 6.4 & 7.6 \\
\hline CB230 & $6^{\prime \prime} 1 \times 4^{\prime \prime} .2 \quad\left(7^{\circ}\right)$ & 5.5 & 4.11 & 4.0 \\
\hline
\end{tabular}

Notes. ${ }^{(a)}$ Stokes I rms noise values come from the maps obtained after self-calibration.

similar fluxes while L1448N-B is four times brighter than L1448N-A.

For five sources, observations sampled baselines above $40 \mathrm{k} \lambda$, i.e., sampling smaller spatial scales and most compact components of the envelope. We produce new maps of these sources using only visibilities that have a radius in the $u v$ plane larger than $40 \mathrm{k} \lambda$. We use a natural weighting scheme to produce the maps in order to achieve the highest point-source sensitivity. These maps are shown in Fig. 4. The flux densities of the compact components are $\sim 150,25,580,530$, and $1120 \mathrm{mJy}$ for L1448N, L1448-2A, NGC 1333 IRAS4A, NGC 1333 IRAS4B, and IRAS16293, respectively; for L1448N and L1448-2A, the compact component only accounts for less than one-tenth of the total flux; and for NGC 1333 IRAS4A and IRAS16293 for one-quarter of the total flux. This means that for these objects most of the mass is still in the large-scale envelope rather than in a massive disk, and that our SMA observations allow us to trace the polarized emission at the envelope scales where most of the mass resides with only a little contamination from a possible central protostellar disk. The visibility profile of NGC 1333 IRAS4B is the flattest in our sample (Fig. 3). One-third of the total $0.87 \mathrm{~mm}$ flux is contained in the compact component. This flat visibility profile is consistent with those analyzed in Looney et al. (2003) and those obtained at both $1 \mathrm{~mm}$ and $3 \mathrm{~mm}$ by the IRAM-PdBI CALYPSO ${ }^{2}$ survey. The profile drops steeply at $u v$ distances larger than $60 \mathrm{k} \lambda$, which suggests that NGC 1333 IRAS4B either has a compact $\left(F W H M<6^{\prime \prime}\right)$ envelope or that its submillimeter emission at scales $3-6^{\prime \prime}$ is dominated by the emission from a large Gaussian disk-like structure. We note that both NGC 1333 IRAS4A and IRAS4B have very compact envelopes (envelope outer radii $\left.<6^{\prime \prime}\right)$ as seen with the PdBI observations at $1 \mathrm{~mm}$ and $3 \mathrm{~mm}$ (Maury et al., in prep).

\subsection{Polarization results}

Figure B.1 shows the maps of the Stokes $Q$ and $U$ parameters and the polarization intensity and polarization fraction maps obtained when combining these maps. The peak polarization intensities and the median polarization fractions (defined as the unweighted ratio of the median polarization intensity to the flux

\footnotetext{
2 http://irfu.cea.fr/Projets/Calypso
}

intensity) are listed in Table 4. In spite of their low luminosity at SMA scales, we detect linearly polarized continuum emission in all the low-mass protostars, even using a conservative $3 \sigma$ threshold. Median polarization fractions range from a few $\%$ for IRAS16293 and L1448N to $10 \%$ for B335. We only obtain a weak $2 \sigma$ detection toward the northeastern companion of SMME/HH797 (around 03:43:57.8; +32:03:11.3). This object was classified as a Class 0 proto-brown dwarf candidate by Palau et al. (2014). When polarization is detected in the center, we observe that the polarization fraction drops toward this center. For HH797, L1448C, L1448-2A, and IRAS03282, we detect polarization in the envelope at distances between 700 and 1600 au from the Stokes $I$ emission peak, but not in the center itself. The absence of polarization could be linked with an averaging effect at high column density in the beam and/or along the line of sight in those objects. We analyze potential causes for depolarization in Sect. 4.1.

\section{Analysis and discussion}

\subsection{Distribution of the polarized emission}

Polarization is detected in all of our maps, but not always toward the peak of dust continuum emission (Stokes I). A constant polarization fraction would predict, on the contrary, that the polarized intensity scales as Stokes I. Yet, polarization holes or depolarization are often observed in the highdensity parts of star-forming molecular cores and protostellar systems (Dotson 1996; Rao et al. 1998; Wolf et al. 2003; Girart et al. 2006; Tang et al. 2013; Hull et al. 2014). We analyze the distribution of both polarized fraction and intensity in this section in order to probe if and where depolarization is observed.

\subsubsection{Variation of the polarized fraction with environment}

For sources in which polarization is detected within the central $5^{\prime \prime}$, we observe a drop in the polarization fraction (Fig. B.1). To quantify this decrease, we analyze how the polarization fraction varies as a function of the $0.87 \mathrm{~mm}$ flux density. We first regenerate our polarization maps with a homogeneous synthesized beam of 5.5" in all our sources, using independent pixels of one-third the synthesized beam $\left(1.8^{\prime \prime}\right)$. IRAS $4 \mathrm{~A}$ and B are not included in this analysis because their dust continuum emission is barely resolved at 5.5". L1448-2A is not included either since polarization is no longer robustly detected at this new resolution. The relation linking $p_{\text {frac }}$ versus the Stokes $I$ flux density in each individual protostellar envelope is shown in Fig. 5 (top). The continuum flux densities are normalized to the peak intensity for each object to allow a direct comparison. We observe a clear depolarization toward inner envelopes with higher Stokes I fluxes and there is a polarization fraction $\propto I^{-0.6}$ for B335 down to $\propto I^{-1.0}$ for $\mathrm{L} 1448 \mathrm{~N}$ or $\mathrm{HH}_{797}{ }^{3}$. Those coefficient are consistent with results from the literature: the polarization fraction has, for instance, been found $\propto I^{-0.6}$ in Bok globules (Henning et al. 2001), $\propto I^{-0.7}-I^{-0.8}$ in dense cores (Matthews \& Wilson 2000), and down to $\propto I^{-0.97}$ in the main core of NGC 2024 FIR 5 (Lai et al. 2002).

Since polarization holes are usually associated with a highdensity medium, we also analyze how the polarization fraction varies as a function of the gas column density $\mathrm{N}\left(\mathrm{H}_{2}\right)$. We assume that the emission at $0.87 \mu \mathrm{m}$ is optically thin. The column den-

\footnotetext{
3 A constant polarized flux gives a polarization fraction $\propto I^{-1}$.
} 
Table 4. $0.87 \mathrm{~mm}$ integrated flux densities, source sizes, polarization intensities, and fractions.

\begin{tabular}{|c|c|c|c|c|c|c|}
\hline \multirow[t]{3}{*}{ Name } & \multicolumn{4}{|c|}{ On the $0.87 \mathrm{~mm}$ reconstructed maps } & \multirow{3}{*}{$\begin{array}{c}p_{\text {frac }}{ }^{c} \\
(\%)\end{array}$} & \multirow{3}{*}{$\begin{array}{c}\text { From the visibility amplitude curve } \\
0.87 \mathrm{~mm} \text { flux density } \\
\text { (Jy) }\end{array}$} \\
\hline & Flux density $^{a}$ & Mass & Peak intensity & Peak $P_{i}^{b}$ & & \\
\hline & $(\mathrm{Jy})$ & $\left(M_{\odot}\right)$ & (Jy/beam) & (mJy/beam) & & \\
\hline B335 & $0.21 \pm 0.04$ & 0.02 & 0.24 & 25.3 & 10.5 & $0.51 \pm 0.05$ \\
\hline SVS13 & $1.40 \pm 0.28$ & 0.29 & 1.06 & 14.8 & 2.5 & $1.54 \pm 0.15^{d}$ \\
\hline HH797 & $0.47 \pm 0.09$ & 0.10 & 0.71 & 5.7 & 3.0 & $0.94 \pm 0.09$ \\
\hline L1448C & $0.57 \pm 0.11$ & 0.11 & 0.65 & 12.5 & - & $1.00 \pm 0.01$ \\
\hline L1448N & $1.50 \pm 0.30$ & 0.31 & 1.34 & 20.3 & 2.7 & $1.93 \pm 0.19^{d}$ \\
\hline L1448-2A & $0.34 \pm 0.07$ & 0.07 & 0.27 & 5.7 & - & $0.48 \pm 0.05$ \\
\hline IRAS03282 & $0.44 \pm 0.09$ & 0.09 & 0.65 & 9.9 & - & $1.17 \pm 0.12$ \\
\hline NGC 1333 IRAS4A & $2.38 \pm 0.48$ & 0.50 & 2.57 & 77.3 & 8.8 & $6.79 \pm 0.68$ \\
\hline NGC 1333 IRAS4B & $1.42 \pm 0.28$ & 0.30 & 2.89 & 14.9 & 4.7 & $4.10 \pm 0.41$ \\
\hline IRAS16293 & $4.81 \pm 0.96$ & 0.26 & 3.83 & 39.2 & 1.7 & $5.96 \pm 0.60^{d}$ \\
\hline L1157 & $0.43 \pm 0.09$ & 0.10 & 0.71 & 31.4 & 4.5 & $1.07 \pm 0.11$ \\
\hline CB230 & $0.22 \pm 0.04$ & 0.09 & 0.37 & 14.3 & 6.4 & $0.58 \pm 0.06$ \\
\hline
\end{tabular}

Notes. ${ }^{(a)}$ Flux density estimated within the central 6000 au for SVS13, 3000 au for other sources. ${ }^{(b)}$ Not all the sources have an intensity peak co-spatial with the polarized intensity peak. ${ }^{(c)}$ Polarization fraction defined as the unweighted ratio of the median polarization to total flux. ${ }^{(d)}$ The visibility data allows us to separate the two components of wide binaries. The fluxes are those of the object located at the phase center, e.g., L1448N-B, SVS13-B and IRAS16293-A.

Table 5. Comparison with SCUBA observations from Di Francesco et al. (2008).

\begin{tabular}{|c|c|c|c|c|c|c|}
\hline \multirow{3}{*}{ Name } & \multicolumn{2}{|r|}{ SCUBA $850 \mu \mathrm{m}$} & \multirow{3}{*}{$\begin{array}{c}\text { Flux density } \\
(\mathrm{Jy})\end{array}$} & \multicolumn{2}{|c|}{ Comparison SMA / SCUBA } & \multirow{3}{*}{$\begin{array}{l}\text { Total flux ratio } \\
\text { SMA/SCUBA }\end{array}$} \\
\hline & Peak intensity & Effective radius & & Rescaled peak intensity ${ }^{a}$ & Peak intensity ratio & \\
\hline & (Jy/beam) & $\left({ }^{\prime \prime}\right)$ & & (Jy/SMA beam) & SMA/SCUBA & \\
\hline B335 & $1.45 \pm 0.14$ & 34.5 & $2.38 \pm 0.36$ & $0.46 \pm 0.07$ & $0.52 \pm 0.09$ & $0.09 \pm 0.02$ \\
\hline HH797 & $1.76 \pm 0.18$ & 43.2 & $4.67 \pm 0.70$ & $0.57 \pm 0.09$ & $1.24 \pm 0.22$ & $0.12 \pm 0.03$ \\
\hline L1448C & $2.37 \pm 0.24$ & 43.5 & $4.15 \pm 0.62$ & $0.77 \pm 0.12$ & $0.84 \pm 0.15$ & $0.14 \pm 0.03$ \\
\hline L1448N & $5.46 \pm 0.55$ & 49.3 & $10.18 \pm 1.53$ & $0.96 \pm 0.14$ & $1.27 \pm 0.23$ & $0.15 \pm 0.04$ \\
\hline L1448-2A & $1.41 \pm 0.14$ & 36.8 & $2.24 \pm 0.34$ & $0.24 \pm 0.04$ & $0.97 \pm 0.17$ & $0.15 \pm 0.04$ \\
\hline IRAS03282 & $1.30 \pm 0.13$ & 45.2 & $2.28 \pm 0.34$ & $0.42 \pm 0.06$ & $1.54 \pm 0.28$ & $0.18 \pm 0.04$ \\
\hline NGC 1333 IRAS4A & $11.4 \pm 0.11$ & 38.5 & $14.4 \pm 2.16$ & $1.40 \pm 0.21$ & $1.83 \pm 0.33$ & $0.17 \pm 0.04$ \\
\hline NGC 1333 IRAS4B & $5.46 \pm 0.55$ & 40.7 & $8.90 \pm 1.34$ & $0.75 \pm 0.11$ & $3.83 \pm 0.69^{b}$ & $0.16 \pm 0.04$ \\
\hline IRAS16293 & $20.2 \pm 0.20$ & 56.8 & $29.5 \pm 4.43$ & $3.43 \pm 0.51$ & $1.12 \pm 0.20$ & $0.16 \pm 0.04$ \\
\hline L1157 & $1.58 \pm 0.16$ & 42.4 & $2.46 \pm 0.37$ & $0.59 \pm 0.09$ & $1.20 \pm 0.22$ & $0.17 \pm 0.04$ \\
\hline CB230 & $1.22 \pm 0.12$ & 43.2 & $2.35 \pm 0.35$ & $0.45 \pm 0.07$ & $0.82 \pm 0.15$ & $0.09 \pm 0.02$ \\
\hline
\end{tabular}

Notes. ${ }^{(a)}$ In order to estimate what the peak intensity of the SCUBA maps would correspond to in a SMA beam, we considered that the envelope follows a $r^{-2}$ density profile, thus that the intensity would scale in $1 / r$. The SMA beam for each source is provided in Table 3. The SCUBA FWHM at $850 \mu \mathrm{m}$ is $14^{\prime \prime}$. SVS13 is not included in the table because SVS13-A and B are not resolved by SCUBA. ${ }^{(b)}$ This high value can be explained by the very flat intensity profile of the source that is barely resolved in our SMA observations (see Fig. 3).

sity is derived using the following formula from Schuller et al. (2009):

$\mathrm{N}\left(\mathrm{H}_{2}\right)=\frac{S_{v}}{\mathcal{D} \mu_{\mathrm{H}_{2}} m_{\mathrm{H}} \Omega \kappa_{v} B_{v}(T)}$,

where $S_{v}$ is the flux density, $\mu_{\mathrm{H}_{2}}$ the molecular weight of the ISM $\left(\mu_{\mathrm{H}_{2}}=2.8\right.$; from Kauffmann et al. 2008), $m_{\mathrm{H}}$ the mass of an hydrogen atom, $\Omega$ the solid angle covered by the beam, $\kappa_{v}$ the dust opacity (tabulated in Ossenkopf \& Henning 1994, $1.85 \mathrm{~cm}^{2} \mathrm{~g}^{-1}$ at $0.87 \mathrm{~mm})$, and $B_{v}(T)$ the Planck function at a dust temperature $T$. The dust-to-gas mass ratio $\mathcal{D}$ is assumed to be 0.01 .

The temperature is not constant throughout the object envelopes. We assume that the temperature profile in optically thin outer envelopes follows $T(r) \propto r^{-0.4}$ and we scale the profiles using the protostellar internal luminosities (Terebey et al. 1993),

$T=38 L_{\text {int }}^{0.2}\left(\frac{r}{100 \mathrm{au}}\right)^{-0.4}$.
Internal luminosities $\mathrm{L}_{\mathrm{int}}$ are calculated from Herschel Gould Belt Survey in Sadavoy et al. (2014) for the Perseus sources and from Maury et al. (in prep) for the other sources. The value $L_{\text {int }}$ is approximated to the bolometric luminosity if no internal luminosity could be found in the literature. Figure 5 (bottom) presents the dependency of $p_{\text {frac }}$ with local column density. Even if the relation observed presents a large scatter, our results confirm that the polarization fraction decreases with increasing column density in the envelopes of our sample.

Along with the polarization fraction variations, the distribution of polarized emission intensities can also help us determine the cause of depolarization. The SCUBAPOL observations have shown that the polarization intensity can decrease at high densities (Crutcher et al. 2004). We do not observe such a trend in our sample. In Fig. 5, the slope of the polarization fraction relation with the dust continuum intensity is close to -1 or flatter for most sources. This means that the polarization intensity is 

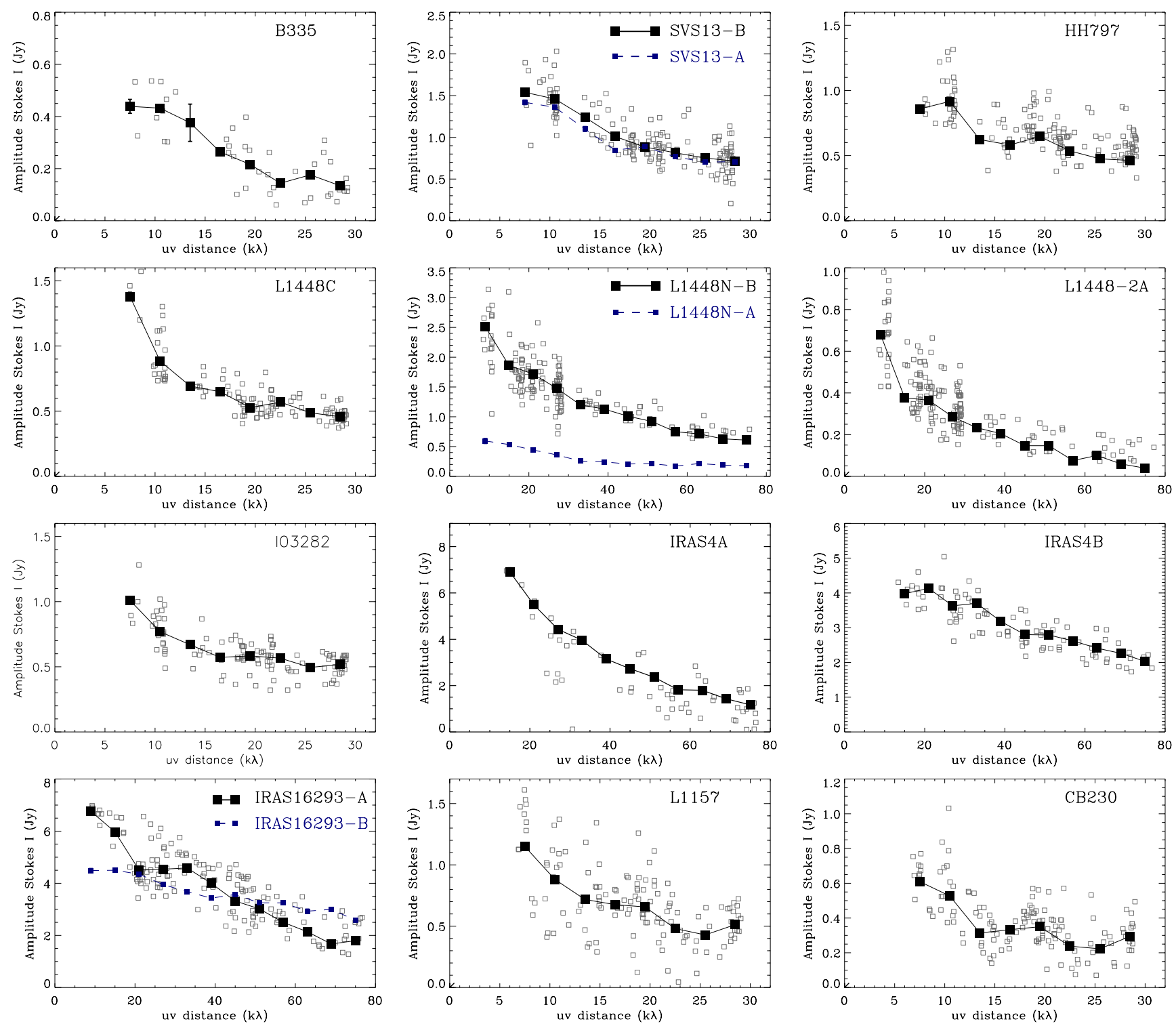

Fig. 3. Stokes $I$ visibility amplitude as a function of $u v$ distance (in units of $\mathrm{k} \lambda$ ). The open squares are the amplitudes of the visibilities channelaveraged and time-averaged every 120s. The black squares are derived averaging these amplitudes of the visibilities in $6 \mathrm{k} \lambda$ bins for L1448N, L1448-2A, NGC 1333 IRAS4A, IRAS4B, and IRAS16293 and $3 \mathrm{k} \lambda$ bins for the other sources. For the wide-binary L1448N, SVS13, and IRAS16293, we model and isolate the two sources separately. For IRAS4B, we also isolate and remove NGC 1333 IRAS4B2 from the visibility data.

constant and even increases (i.e., B335, SVS13, or IRAS16293) toward the central regions (see also the polarization intensity and fraction maps provided in Fig. B.1). These results suggest that for these sources, the decrease of the polarization fraction in the center is not linked to a full depolarization, but could indicate a variation in the polarization efficiency itself (see next section).

\subsubsection{Potential causes for depolarization}

Observations and models or simulations (Padoan et al. 2001; Bethell et al. 2007; Falceta-Gonçalves et al. 2008; Kataoka et al. 2012) have shown that depolarization can be linked with both geometrical effects (e.g., averaging effects linked with the complex structure of the magnetic field along the line of sight) and physical effects (e.g., collisional/mechanical disalignment, lower grain alignment efficiency, and variation of the grain population).
We discuss here some of these depolarization effects. We note that our sample is sensitivity limited, which means that there is a potential bias toward strong polarization intensities. We also note that the polarized emission presented in this paper comes from the envelope and that we are not sampling the polarized (or unpolarized) emission from the inner $(<500 \mathrm{au})$ envelope.

Geometrical effects. Our SMA observations recover polarized emission from previously reported polarization holes observed with single-dish observations. Observations of B335 and CB230 at a 2200 au resolution using SCUBAPOL (Holland et al. 1999) performed by Wolf et al. (2003) show that the polarization fraction decreases from 6-15\% in the outer parts of the cores to a few $\%$, thus at scales in which $3-10 \%$ polarization is detected with the SMA (see Fig. 6 for an illustration in CB230). The central depolarization observed by Wolf et al. (2003) in B335 and CB230 is thus partially due to beam averaging effects or 

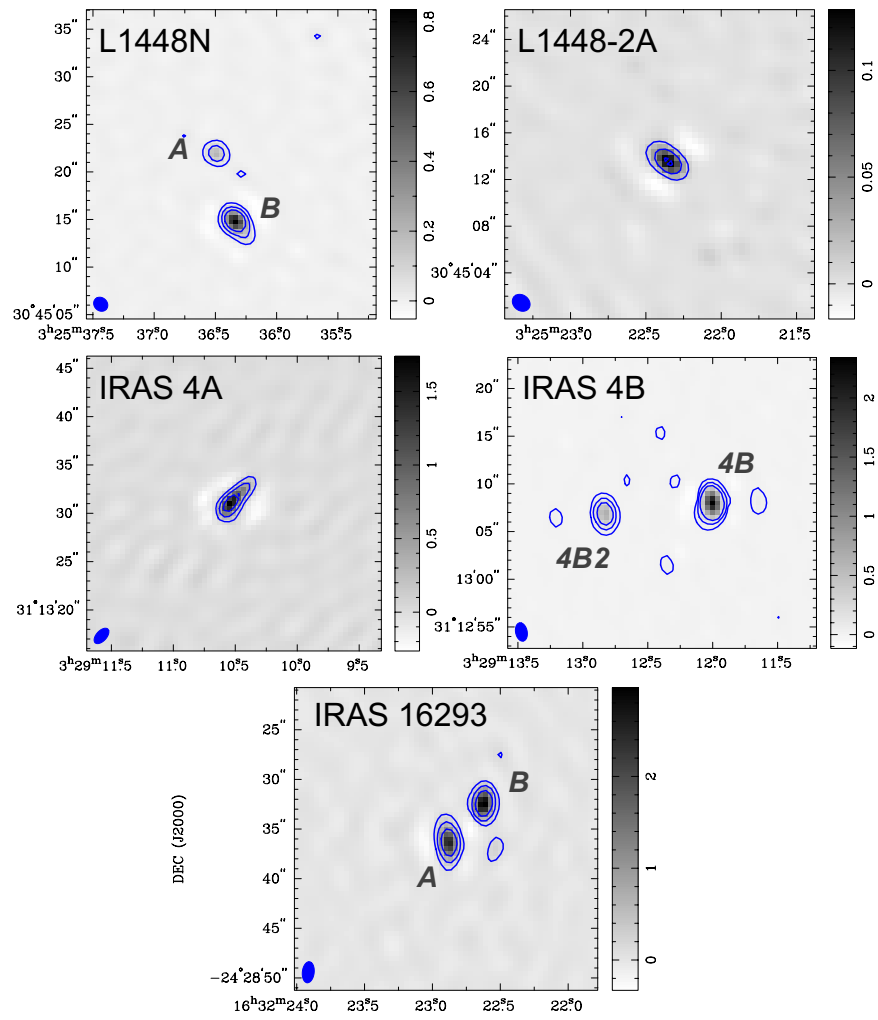

Fig. 4. $345 \mathrm{GHz}$ dust continuum emission in L1448N, L1448-2A, NGC 1333 IRAS4A, NGC 1333 IRAS4B, and IRAS16293 when only visibilities above $40 \mathrm{k} \lambda$ are kept. We apply a natural weighting to produce these maps. Contours at $[5,20,50] \sigma$ appear in blue. The beam sizes are $\sim 1$ '” $9 \times 1$ '! 2 .

mixing of the polarized signal along the line of sight, an envelope pattern that our SMA resolution now allows us to recover. Recent polarization observations of B335 with ALMA at even higher resolution by Maury et al. (2018) have confirmed that polarization is still detected toward the center of the B335 envelope at $0.5-5^{\prime \prime}$ scales, which is a sign that the dust emission stays polarized $(\mathrm{p} \sim 3-7 \%)$ at small scales in high-density regions.

Optical depth effects. As already reported in Girart et al. (2006), a two-lobe distribution of the polarization intensity is detected toward NGC 1333 IRAS4A. We report a similar two-lobe structure for the first time in NGC 1333 IRAS4B and detect peaks of polarization intensity on each side of the north-south outflow. Liu et al. (2016) have found that the polarization intensity of NGC 1333 IRAS4A observed at $6.9 \mathrm{~mm}$ has a more typical distribution in which the polarization intensity peaks toward the source center; these authors have attributed this variation of the polarization distribution with wavelength to optical depth effects. Our temperature brightnesses rather suggest that the $0.87 \mathrm{~mm}$ emission is optically thin at the envelope scales probed in this paper, for all our objects. We note that modeling the magnetic field in NGC 1333 IRAS4A, Gonçalves et al. (2008) showed that strong central concentrations of magnetic field lines could reproduce a two-lobe structure in the polarized intensity distribution.

Grain alignment, grain growth. Several studies of dense molecular clouds or starless cores have shown that the slope of the polarization degree can reach values of -1 (as in some of our objects) or lower with respect to $A_{\mathrm{V}}$ or $I / I_{\max }$ and interpret this slope
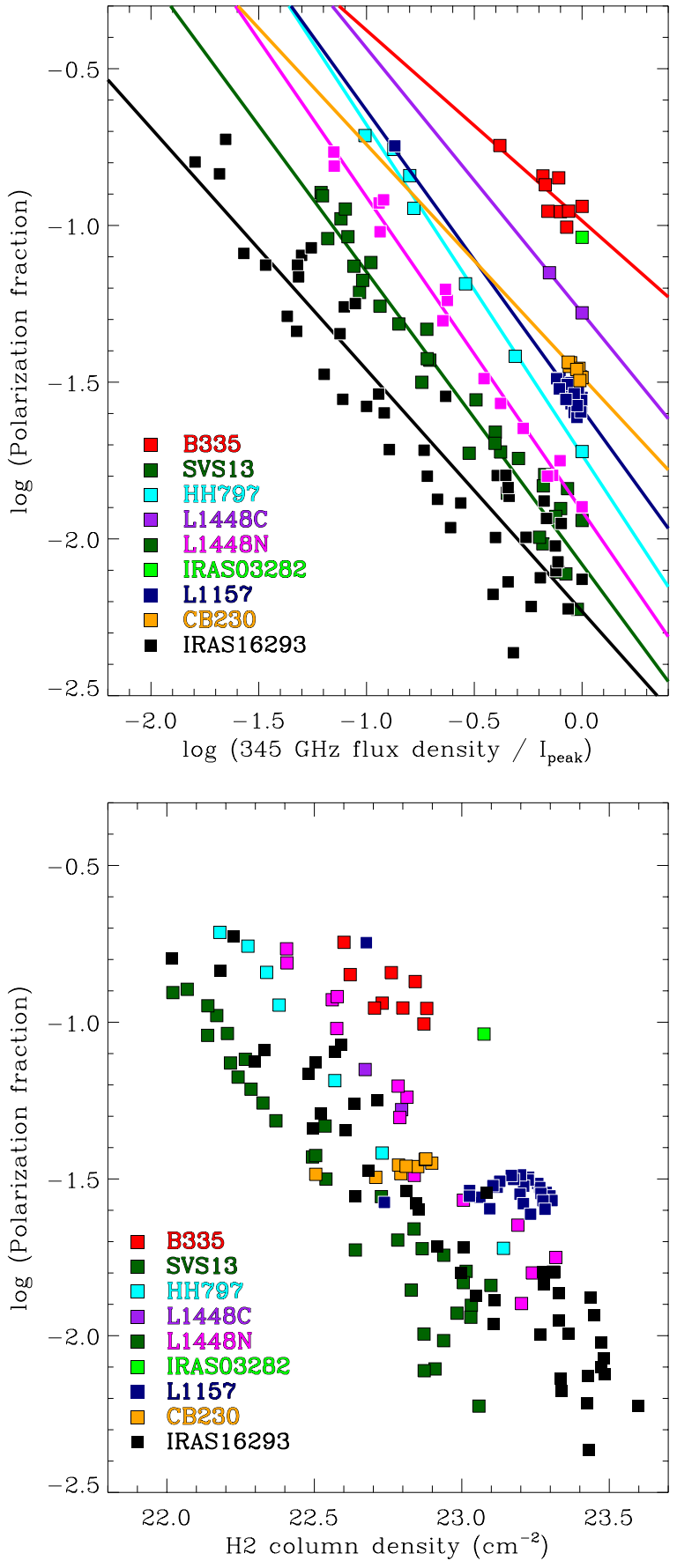

Fig. 5. Polarization fractions as a function of Stokes $I$ continuum intensities at $0.87 \mathrm{~mm}$ (top panel) or as a function of the $\mathrm{H}_{2}$ column densities (bottom panel). Maps have been regenerated to share a common synthesized beam of about 5.5" and rebinned to have a grid with a common pixel size of $1.8^{\prime \prime}$. The continuum flux densities plotted in the top panel are in units of Jy/beam but are normalized to the peak intensity for each object. The solid lines are the best fit to the function $p_{\text {frac }} \propto\left(I / I_{\max }\right)^{a}$. The $\mathrm{H}_{2}$ column densities plotted in the bottom panel are in units of $\mathrm{cm}^{-2}$ and are derived using Eqs. (5) and (6).

as a sign of a decrease or absence of grain alignment at higher densities (Alves et al. 2014; Jones et al. 2015, 2016). Even if this is not the case in this work because our objects all have a central heating source, the degree of polarization we detect is still extremely sensitive to the efficiency of grain alignment, which is itself very sensitive to the grain size distribution 


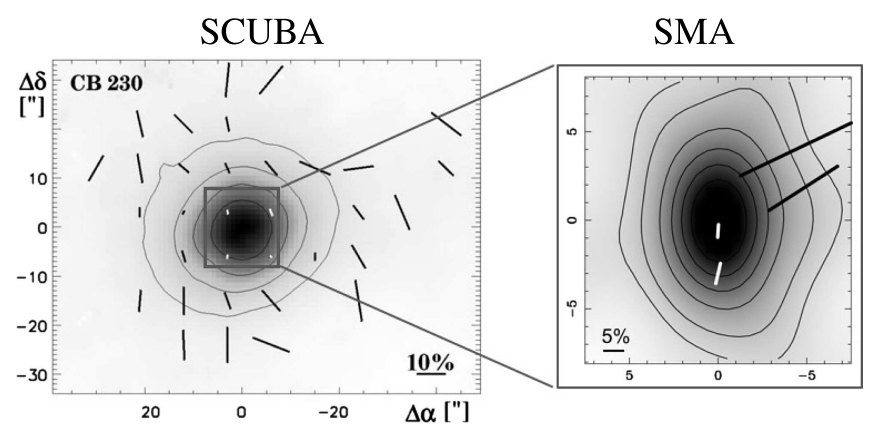

Fig. 6. Left: SCUBA $0.85 \mathrm{~mm}$ continuum maps with polarization orientation overlaid from Wolf et al. (2003). The contour lines indicate 20\%, $40 \%, 60 \%$, and $80 \%$ of the maximum intensity. Right: zoom-in into the scales covered with the SMA at $0.87 \mathrm{~mm}$. The contours are the same than in Fig. 2. For both, the bar length is proportional to the polarization fraction.

and potential grain growth (Bethell et al. 2007; Pelkonen et al. 2009; Brauer et al. 2016). This grain growth has already been suggested in Class 0 protostars such as L1448-2A or L1157 (Kwon et al. 2009), in which there is a faster grain growth toward the central regions. More observations combining various submillimeter wavelengths at various scales will help us probe the dust spectral index throughout the envelope, analyze the scales at which grain growth is expected (Chacón-Tanarro et al. 2017), probe the various environmental dependencies of the grain alignment efficiency (Whittet et al. 2008), and observationally constrain the dependence on the polarization degree and the grain size as predicted for instance by radiative torque models (see Bethell et al. 2007; Hoang \& Lazarian 2009; Andersson et al. 2015; Jones et al. 2015).

\subsection{Orientation of the magnetic field}

The B-field lines are inferred from the polarization angles by applying a $90^{\circ}$ rotation. The mean magnetic field orientations in the central 1000 au are provided in Table 6 . The B orientation is overlaid with red bars in Fig. 2. We also show the $>2 \sigma$ detections (orange bars). Although these are subject to higher uncertainties we stress that they are mostly consistent with the neighboring $3 \sigma$ detection line segments. For robustness, however, these lower significance detections are not used in this analysis. Under fluxfreezing conditions, the pull of field lines in strong gravitational potentials is expected to create an hourglass morphology of the magnetic field lines, centered around the dominant infall direction, during protostellar collapse. Using CARMA observations, Stephens et al. (2013) showed that in L1157, the full hourglass morphology becomes apparent around 550 au. Using SMA observations, Girart et al. (2006) also observed this hourglass shape in NGC 1333 IRAS4A. The resolution we select for this analysis of B at envelope scale is not sufficient to reveal the hourglass morphology in most of our objects. Only one of our sources, L1448-2A, shows hints of an hourglass shape at envelope scales (see Fig. 2) but the nondetection of polarization in the northern quadrant does not allow us to detect a full hourglass pattern in this source. Better sensitivity observations are needed to confirm this partial detection.

\subsubsection{Large scales versus small scales}

Our B-field orientations can be compared to SHARP $350 \mu \mathrm{m}$ and SCUBAPOL $850 \mu \mathrm{m}$ polarization observations that provide the orientation of the magnetic field at the surrounding cloud scales for half of our sample. We use the SHARP results presented in Attard et al. (2009), Davidson et al. (2011), and Chapman et al. (2013) along with the SCUBAPOL of Wolf et al. (2003) and Matthews et al. (2009). The large-scale and smaller scale B orientations match for NGC 1333 IRAS4A, L1448N, L1157, CB230, and HH797. The SMA B orientation in L1448$2 \mathrm{~A}$ is difficult to reconcile with that detected with SHARP. In B335, the SCUBA observations seem also inconsistent (northsouth direction) with the SMA B orientation. However, only two significant line segments are robustly detected with SCUBA and Bertrang et al. (2014) detected a mostly poloidal field in the B335 core using near-infrared polarization observations at 10000 au scales. Recent observations of B335 with ALMA have revealed a very ordered topology of the magnetic field structure at $50 \mathrm{au}$, which has a combination of a large-scale poloidal magnetic field (outflow direction) and a strongly pinched magnetic field in the equatorial direction (Maury et al. 2018). Our SMA observations suggest that above $>1000$ au scales, the poloidal component dominates in B335. All these results show that an ordered B morphology from the cloud to the envelope is observed for most of our objects. Our results also seem to confirm that sources with consistent large- to small-scale fields (e.g., B335, NGC 1333 IRAS4A, L1157, and CB230) tend to have a high polarization fraction, similar to that found in Hull et al. (2013).

\subsubsection{Variation of the $B$ orientation with wavelength}

Only a few studies have investigated the relationship between the magnetic field orientation and wavelength on similar scales. Using single-dish observations, Poidevin et al. (2010) found that in star-forming molecular clouds, most of the $0.35 \mathrm{~mm}$ and $0.85 \mathrm{~mm}$ polarization data had a similar polarization pattern. In Fig. 7, we compare the $0.87 \mathrm{~mm}$ SMA B-field orientation to those observed at $1.3 \mathrm{~mm}$ by Stephens et al. (2013) and Hull et al. (2014). The CARMA maps are rebinned to match the grid of our SMA maps. Seven objects are common to the two samples and have co-spatial detections. We do not compare the SMA and CARMA results for B335 and L1448C, as polarization is only marginally $(<3.5 \sigma)$ detected at $1.3 \mathrm{~mm}$ (Hull et al. 2014). The orientations at 0.87 and $1.3 \mathrm{~mm}$ match for L1157, SVS13, L1448N-B, NGC 1333 IRAS4A, and IRAS4B. For SVS13-B, the orientations only slightly deviate in the southeast part, but the position angles remain consistent within the uncertainties added in quadrature ( $8^{\circ}$ uncertainties for SMA, $14^{\circ}$ for CARMA). In L1448N-B, the orientation of the B-field, perpendicular to the outflow, is also consistent with BIMA (Berkeley Illinois Maryland Array; Welch et al. 1996) $1.3 \mathrm{~mm}$ observations presented in Kwon et al. (2006). The orientations at $0.87 \mathrm{~mm}$ and $1.3 \mathrm{~mm}$ deviate in the outer parts of the envelope in NGC 1333 IRAS4B, in regions with low detected polarized intensities. In CB230, the $3 \sigma$ detection at $0.87 \mathrm{~mm}$ and $1.3 \mathrm{~mm}$ do not exactly overlap physically but the east-west orientation of our central detections is consistent with the orientation found with CARMA by Hull et al. (2014). Finally, for L1448-2A the SMA $0.87 \mathrm{~mm}$ and CARMA $1.3 \mathrm{~mm}$ detections do not overlap, making a direct comparison between wavelength difficult for this object. We note that the flat structure of the dust continuum emission oriented northeast-southwest observed at $0.87 \mathrm{~mm}$ is consistent with that observed at $1.3 \mathrm{~mm}$ by Yen et al. (2015). The $1.3 \mathrm{~mm}$ observations from TADPOL are not in agreement: a northwest-southeast extension is observed along the outflow and the polarization detections (blue bars in Fig. 7) from TADPOL in the central part of L1448-2A might be suffering from contamination by CO polarized emission from the outflow itself. Their $2.5 \sigma$ detections 

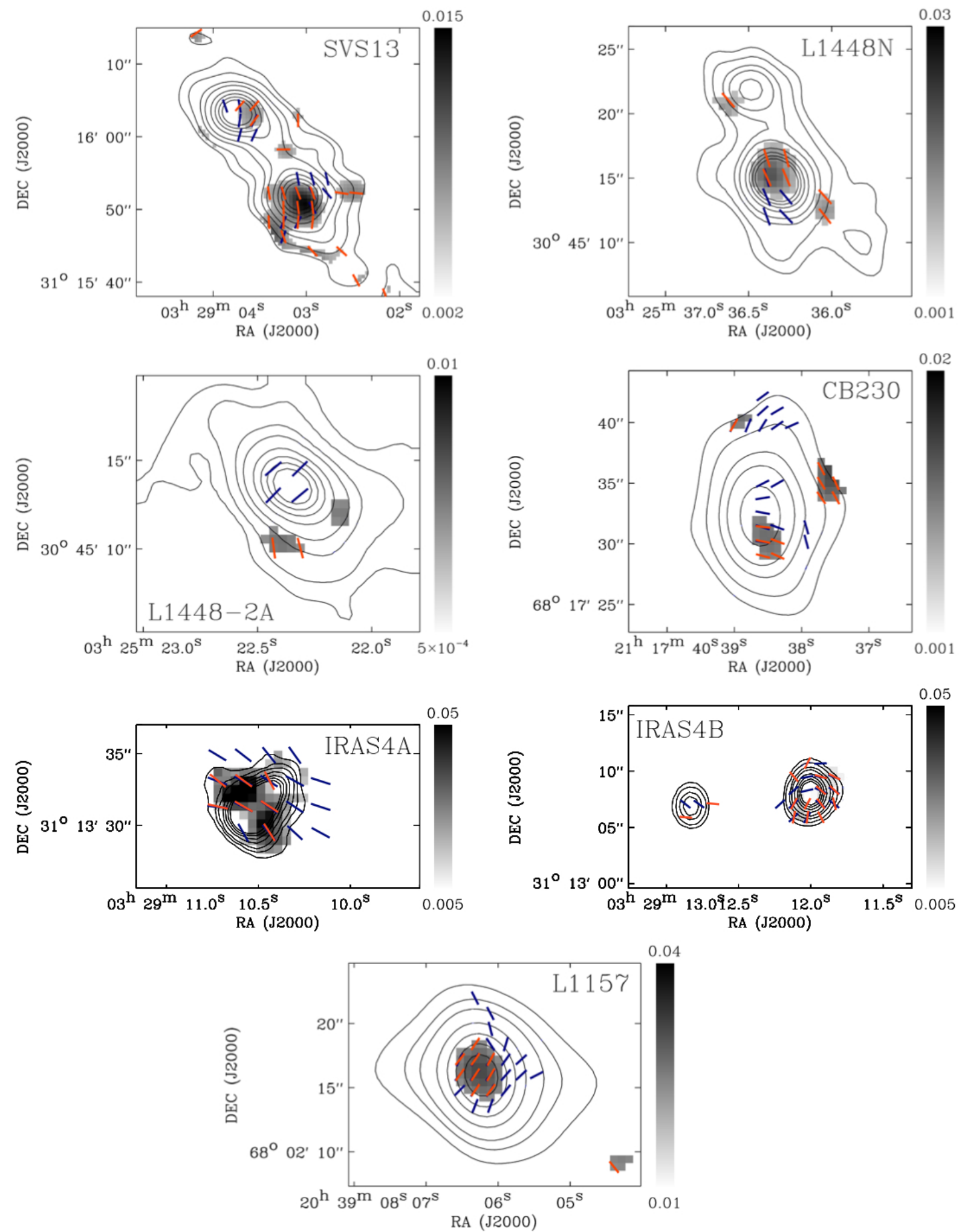

Fig. 7. B-field orientation derived from SMA $0.87 \mathrm{~mm}$ observations in red ( $3 \sigma$ detections) and from CARMA $1.3 \mathrm{~mm}$ observations in blue ( $3.5 \sigma$ detections from Hull et al. 2014). The underlying map is the polarization intensity in mJy/beam and contours are the Stokes $I$ continuum emission (same levels as in Fig. 2).

along the equatorial plane are, however, consistent with our $2 \sigma$ detections. Our conclusion is that the orientations observed at $0.87 \mathrm{~mm}$ and $1.3 \mathrm{~mm}$ are consistent. Because those two wavelengths are close to each other, they probably trace optical depths on which the magnetic field orientations stay ordered.

\subsubsection{Misalignment with the outflow orientation}

Whether or not the rotation axis of protostellar cores are aligned with the main magnetic field direction is still an open question. From an observational point of view, it remains difficult to 
precisely pinpoint the rotation axis of protostellar cores. The outflow axis is often used as a proxy for this key parameter, as protostellar bipolar outflows are believed to be driven by hydromagnetic winds in the circumstellar disk (Pudritz \& Norman 1983; Shu et al. 2000; Bally 2016). The core rotation and outflow axes are thus usually considered as aligned ${ }^{4}$. Synthetic observations of magnetic fields in protostellar cores have shown that magnetized cores have strong alignments of the outflow axis with the $\mathrm{B}$ orientation while less magnetized cores present more random alignment (Lee et al. 2017). The magnetohydrodynamic (MHD) collapse models predict in particular that magnetic braking should be less effective if the envelope rotation axis and magnetic field are not aligned (Hennebelle \& Ciardi 2009; Joos et al. 2012; Krumholz et al. 2013; Li et al. 2013; Seifried et al. 2015). The comparison between the rotation axis and B-field orientation is thus a key to understand the role of $\mathrm{B}$ in regulating the collapse. Previous observational works found that outflows do not seem to show a preferential direction with respect to the magnetic field direction both on large scales (Curran \& Chrysostomou 2007) and at 1000 au scales (Hull et al. 2013). A similar absence of correlation is also reported in a sample of high-mass star-forming regions by Zhang et al. (2014). Our detections of the main B-field component at envelope scales for all the protostars of our sample allow us to push the analysis further for low-mass Class 0 objects.

The misalignments between the $\mathrm{B}$ orientation and the outflow axis driven by the protostars can be observed directly from Fig. 2. Table 6 provides the projected position angle of the outflows as well as the angle difference between this outflow axis and the main envelope magnetic field orientation when $B$ is detected in the central region. For half of the objects, the magnetic field lines are oriented within $40^{\circ}$ of the outflow axis but some sources show a rather large $\left(>60^{\circ}\right)$ difference of angles (e.g., L1448N-B and CB230). Using the maps generated with a common 5.5" synthesized beam (thus excluding NGC 1333 IRAS 4A, 4B and L1448-2A; see $\S 4.1$ ), we build a histogram of the projected angles between the magnetic field orientation and outflow direction (hereafter $\mathcal{A}_{\mathcal{B}-O}$ ) shown in Fig. 8. We also compare the full histogram with that restricted to detections within the central 1500 au (Fig. 8, bottom panel). The distribution of $\mathcal{A}_{\mathcal{B}-O}$ looks roughly bimodal, suggesting that at the scales traced with the SMA, the B-field lines are either aligned or perpendicular to the outflow direction. Hull et al. (2014) found hints that for objects with low polarization fractions, the B-field orientations tend to be preferentially perpendicular to the outflow. In our sample focusing on solar-type Class 0 protostars, we do not observe a relation between $\mathcal{A}_{\mathcal{B}-O}$ and the polarization fraction nor intensity. For instance, L1448N-B and SVS13-B have very similar polarization fractions but very different $\mathcal{A}_{\mathcal{B}-O}$. In the same way, the two Bok globules B335 and CB230 both have a high polarization fraction but exhibit very different $\mathcal{A}_{\mathcal{B}-O}$.

\subsubsection{Relation between misaligned magnetic field, strong rotation, and fragmentation?}

Details concerning the various velocity gradients (orientation, strength) measured in our sources are provided in the Appendix.

\footnotetext{
4 We note that magnetohydrodynamic simulations have shown that the outflowing gas tends to follow the magnetic field lines when reaching scales of a few thousands au even when the rotation axis is not aligned with the large-scale magnetic field. Hence one should always be careful when assuming that the orientation of the outflow traces the rotation axis.
}

Velocity fields in protostars can trace many processes (e.g., turbulence and outflow motions). In particular, gradients detected in the equatorial plane of the envelope can trace both rotation and infall motions. Organized velocity gradients with radius are often interpreted as a sign that the rotational motions dominate the velocity field. In this section, we compare the B orientation and the misalignment $\mathcal{A}_{\mathcal{B}-O}$ with velocity gradients associated with rotation in our envelopes.

$\mathcal{A}_{\mathcal{B}-O}>45^{\circ}$ case. $\mathrm{L} 1448 \mathrm{~N}-\mathrm{B}$ and CB230 have a $\mathcal{A}_{\mathcal{B}-O}$ close to $90^{\circ}$. They both have large envelope masses and velocity gradients perpendicular to their outflow direction detected at hundreds of au scales. A high rotational to magnetic energy could lead to a twist of the field lines in the main rotation plane. Their high mass-to-flux ratio $\mu=M / \Phi \sim E_{\text {grav }} / E_{\text {mag }}$ could also favor a gravitational pull of the equatorial field lines. Both scenarios can efficiently produce a toroidal/radial field from an initially poloidal field that would explain the main field component we observe perpendicular to the outflow/rotation axis. The opposite causal relationship is also possible. An initially misaligned magnetic field configuration could be less efficient at braking the rotation and lead to the large rotational motions detected at envelope scales. Additional observations of Class 0 from the literature show similar misalignments. In NGC 1333 IRAS2A, the B direction is misaligned with the outflow axis (Hull et al. 2014) but aligned with the velocity gradient observed in the combined PdBI+IRAM30 $\mathrm{C}^{18} \mathrm{O}$ map by Gaudel et al. (in prep). In L1527, $\mathcal{A}_{\mathcal{B}-O}$ is nearly $90^{\circ}$ (Segura-Cox et al. 2015) but the B direction follows the clear north-south velocity gradient tracing the Keplerian rotation of a rather large $\sim 60$ au disk (Ohashi et al. 2014). We stress, however, that polarization in disks could also be produced by the self-scattering of dust grains (Kataoka et al. 2015) and not tracing B. Finally, our source NGC 1333 IRAS4A has a B orientation misaligned compared to the small scale northsouth outflow direction (see also Girart et al. 2006) but aligned with the large-scale 45 deg outflow direction (bended outflow; see Appendix). This is consistent with the position angle of the velocity gradient found by Belloche et al. (2006), even if the interpretation of gradients is difficult in IRAS4A because strong infall motions probably dominate the velocity field.

$\mathcal{A}_{\mathcal{B}-O}<45^{\circ}$ case. We find only small misalignments in L1157 and B335, which are sources that show low to no velocity gradient at 1000 au scales (Tobin et al. 2011; Yen et al. 2010; Gaudel et al. in prep). The B orientation of the off-center detection in L1448C (see Fig. 2) is also aligned with the outflow axis. The source has one of the smallest velocity gradient perpendicular to the outflow axis determined by Yen et al. (2015). Our widemultiple systems IRAS16293 (A and B) and SVS13 (A and B) present good alignments. IRAS16293-B does not show rotation but the source is face-on, which might explain the absence of rotation signatures. In IRAS16293-A, the magnetic field has an hourglass shape at smaller scales (Rao et al. 2009), which is a signature of strong magnetic fields in the source; but the source also has a strong velocity gradient perpendicular to the outflow direction that could be responsible for some of the misalignment. Finally, the B direction is slightly tilted in the center of SVS13B. We note that Chen et al. (2007) detected an extended velocity gradient detected along the SVS13-A/SVS13-B axis across the whole SVS13 system, which has been interpreted as core rotation.

Even if our sample is limited, the coincidence of a misalignment of B with the outflow direction when large perpendicular velocity gradients are present or the alignment of $\mathrm{B}$ with the velocity gradient itself strongly suggests that the orientation of $\mathrm{B}$ 
Table 6. Misalignment between the outflow angles $\chi_{o}$ and the mean magnetic field orientation $\chi_{B_{1000}}$ in the central 1000 au.

\begin{tabular}{lccc}
\hline \hline Name & $\begin{array}{c}\chi_{o}^{a} \\
\left(^{\circ}\right)\end{array}$ & $\begin{array}{c}\chi_{B_{1000}} \\
\left(^{\circ}\right)\end{array}$ & $\left|\chi_{o}-\chi_{B_{1000}}\right|$ \\
\hline B335 & 90 & $55 \pm 3$ & 35 \\
SVS13-A & 148 & $140 \pm 3$ & 8 \\
SVS13-B & 160 & $19 \pm 6$ & 39 \\
HH797 & 150 & $113 \pm 2$ & 37 \\
L1448C & 161 & - & - \\
L1448N-B & 105 & $23 \pm 5$ & 82 \\
L1448-2A & 138 & - & - \\
IRAS03282 & 120 & - & - \\
NGC 1333 IRAS4A & 170 & $57 \pm 13$ & 67 \\
NGC 1333 IRAS4B & 0 & $58 \pm 49$ & 58 \\
IRAS16293-A & 145 & $163 \pm 15$ & 18 \\
IRAS16293-B & 130 & $114 \pm 17$ & 16 \\
L1157 & 146 & $146 \pm 4$ & 0 \\
CB230 & 172 & $86 \pm 3$ & 86 \\
\hline
\end{tabular}

Notes. ${ }^{(a)}$ Position angles are provided east of north.

References. Bachiller et al. (1998), Bachiller et al. (2001), Choi (2001), Hull et al. (2013), Hull et al. (2014), Rodríguez et al. (1997), Tafalla et al. (2006), Rao et al. (2009), Santangelo et al. (2015), and Yen et al. (2015).

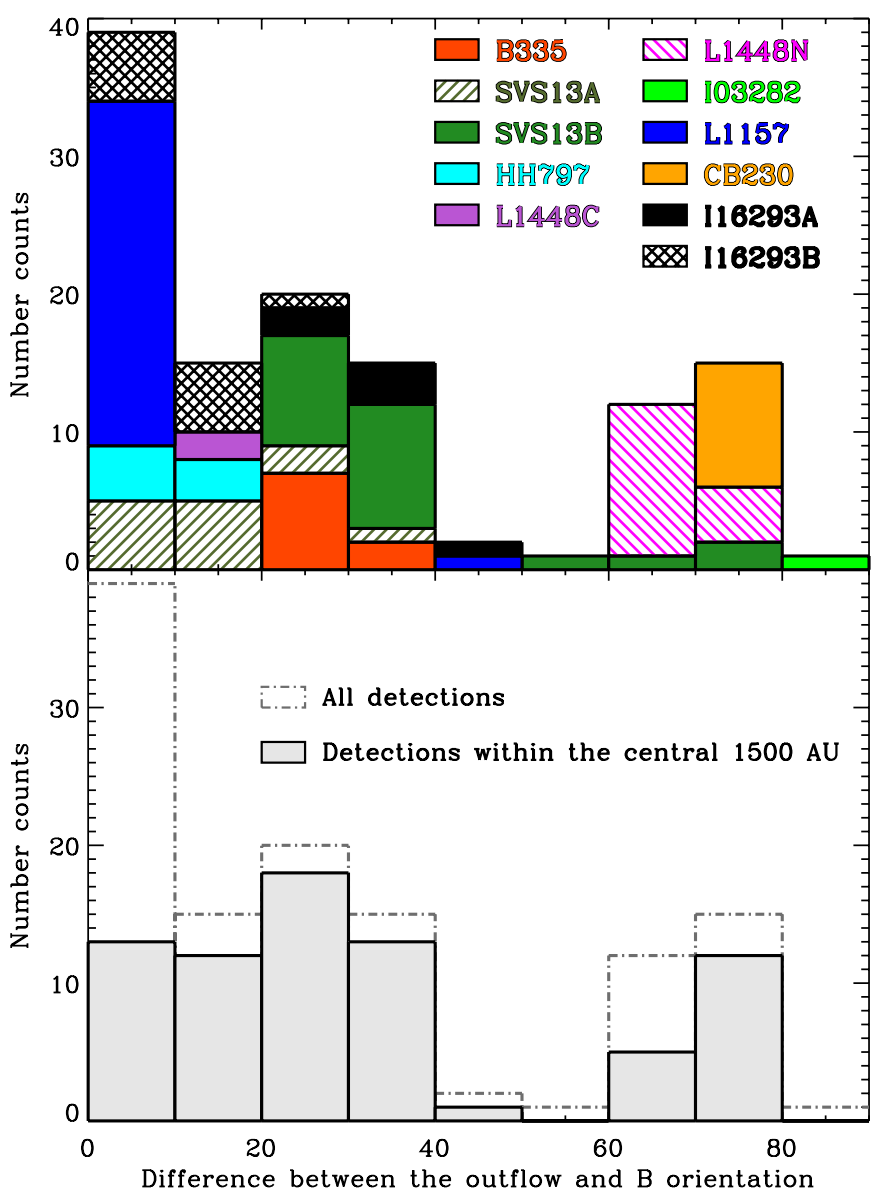

Fig. 8. Histogram of the projected angles between the magnetic field and outflow direction. The data share a common synthesized beam size of about 5.5" and are binned with pixels of $1.8^{\prime \prime}$ for this analysis. The top panel shows the histogram color-coded per source while the bottom panel shows the global histogram (in white) with the histogram restricted to detections within the central 1500 au overlaid in gray. at the envelope scales traced by the SMA can be affected by the strong rotational energy in the envelope. These results are consistent with predictions from numerical simulations that show a strong relation between the angular velocity of the envelope and magnetic field direction (Machida et al. 2005, 2007). Rotationally twisted magnetic fields have already been observed at larger scales (Girart et al. 2013; Qiu et al. 2013). After a given number of periods, rotation could be distorting or even twisting the field lines, producing a toroidal component in the equatorial plane. Using MHD simulations, Ciardi \& Hennebelle (2010) and Joos et al. (2012) showed that the mass ejection via outflows is less efficient when the rotation axis and $\mathrm{B}$ direction are misaligned and that outflows could be less efficient when the rotation axis and $\mathrm{B}$ direction are misaligned. The presence of outflows in sources with $\mathcal{A}_{\mathcal{B}-O} \sim 90^{\circ}$ might favor a twisted/pinched magnetic field scenario compared to an initially misaligned magnetic field configuration.

Reinforcing the possible correlation between the field misalignment and the presence of strong rotational motions, our SMA observations also suggest that a possible correlation could be present between the envelope-scale main magnetic field direction and the multiplicity observed in the inner envelopes. Indeed, sources that present a strong misalignment (IRAS03282, NGC 1333 IRAS4A, L1448N, L1448-2A, or CB230) are all close multiples (Maury et al., in prep.; Launhardt 2004; Chandler et al. 2005; Choi 2005; Tobin et al. 2013, 2016). SVS13-A does host a close multiple but it is a Class I. We are thus not witnessing the initial conditions leading to fragmentation, and the magnetic field we observe could have been severely affected by the evolution of both the environment and the protostar itself. On the contrary, sources that show an alignment between the B direction and the outflow direction (e.g., L1157, B335, SVS13-B, and L1448C) have been studied with ALMA or the NOrthern Extended Millimeter Array (NOEMA) highresolution observations and stand as robust single protostars at envelope scales of 5000-10000 au. No large disk has been detected so far in these sources (Saito et al. 1999; Yen et al. 2010; Chiang et al. 2012; Tobin et al. 2015) while L1448N-B for instance was suggested recently to harbor a large disk encompassing several multiple sources at scales $\sim 100$ au (Tobin et al. 2016). These results support those of Zhang et al. (2014): the possible link between fragmentation and magnetic field topology points toward a less efficient magnetic braking and redistribution of angular momentum when the B-field is misaligned.

\section{Summary}

We perform a survey of $0.87 \mathrm{~mm}$ continuum and polarized emission from dust toward 12 low-mass Class 0 protostars.

(i) By comparing the $0.87 \mathrm{~mm}$ SMA continuum fluxes with single-dish observations, we find that our interferometric observations recover $<20 \%$ of the total fluxes. The fluxes derived directly from the visibility data are also about twice larger than those derived on the reconstructed maps. For five objects, we observed baselines above $40 \mathrm{k} \lambda$ that allow us to separate the most compact components. The low flux fractions contained in the compact component are consistent with the classification as Class 0 of our sources.

(ii) We report the detection of linearly polarized dust emission in all the objects of the sample with mean polarization fractions ranging from $2 \%$ to $10 \%$.

(iii) We find a decrease of the polarization fraction with the estimated $\mathrm{H}_{2}$ column density. Our polarization intensity profiles are 
relatively flat in most of our sources and increases toward the center in B335, SVS13, or IRAS16293, suggesting a decrease in the dust alignment efficiency rather than a polarization cancellation in these sources. Two-lobe structures in the polarized intensity distributions are observed in NGC 1333 IRAS4A and IRAS4B.

(iv) The orientations observed at $0.87 \mathrm{~mm}$ with SMA and $1.3 \mathrm{~mm}$ with CARMA are consistent with each other on the $1000 \mathrm{au}$ scales probed in this analysis.

(v) As in Hull et al. (2013), we find that sources with a high polarization fraction have consistent large-to-small-scale fields. We do not observe, however, a relation between the misalignment of the magnetic field orientation with respect to the outflow axis and the polarization intensity or fraction.

(vi) We find clues that large misalignment of the magnetic field orientation with the outflow orientation could be found preferentially in protostars with a higher rotational energy. The misalignment of $90^{\circ}$ observed in some objects could thus be a signature of winding of the magnetic field lines in the equatorial plane when the rotational energy is significant. Strengthening this possible link, there are also hints that a B-outflow misalignment is found preferentially in protostars that are close multiple and/or harbor a larger disk, while single objects seem to show a good agreement between the magnetic field direction at envelope scales and the direction of their protostellar outflow. This suggests that the topology and strength of magnetic fields at envelope scale may significantly impact the outcome of protostellar collapse, eventually playing a major role in the formation of disks and multiple systems.

Our results are aligned with previous studies of B in massive cores (Zhang et al. 2014) as well as with theoretical predictions from magnetic collapse models (Machida et al. 2005; Joos et al. 2012). More observations of the magnetic field of low-mass protostars at envelope scale would be necessary to observationally confirm these tentative, but promising results and reinforce our interpretations.

Acknowledgements. We thank Baobab Liu for fruitful discussions on this project and Bilal Ladjelate for providing the internal luminosities from the Herschel Gould Belt Survey dataset for the sources belonging to the CALYPSO sample. This project has received funding from the European Research Council (ERC) under the European Union Horizon 2020 research and innovation program (MagneticYSOs project, grant agreement $\mathrm{N}^{\circ}$ 679937). JMG is supported by the MINECO (Spain) AYA2014-57369-C3 and AYA2017-84390-C2 grants. SPL acknowledges support from the Ministry of Science and Technology of Taiwan with Grant MOST 106-2119-M-007-021-MY3. This publication is based on data of the Submillimeter Array. The SMA is a joint project between the Smithsonian Astrophysical Observatory and the Academia Sinica Institute of Astronomy and Astrophysics, and is funded by the Smithsonian Institution and the Academia Sinica.

\section{References}

Alves, F. O., Frau, P., Girart, J. M., et al. 2014, A\&A, 569, L1

Andersson, B.-G., Lazarian, A., \& Vaillancourt, J. E. 2015, ARA\&A, 53, 501

Andre, P., Ward-Thompson, D., \& Barsony, M. 1993, ApJ, 406, 122

Andre, P., Ward-Thompson, D., \& Barsony, M. 2000, Protostars and Planets IV, 59

Attard, M., Houde, M., Novak, G., et al. 2009, ApJ, 702, 1584

Bachiller, R., Martin-Pintado, J., \& Planesas, P. 1991, A\&A, 251, 639

Bachiller, R., Guilloteau, S., Dutrey, A., Planesas, P., \& Martin-Pintado, J. 1995, A\&A, 299, 857

Bachiller, R., Guilloteau, S., Gueth, F., et al. 1998, A\&A, 339, L49

Bachiller, R., Gueth, F., Guilloteau, S., Tafalla, M., \& Dutrey, A. 2000, A\&A, 362, L33

Bachiller, R., Pérez Gutiérrez, M., Kumar, M. S. N., \& Tafalla, M. 2001, A\&A, 372,899

Bally, J. 2016, ARA\&A, 54, 491
Barsony, M., Ward-Thompson, D., André, P., \& O’Linger, J. 1998, ApJ, 509, 733

Belloche, A. 2013, in EAS Pub. Ser., eds. P. Hennebelle, \& C. Charbonnel, 62, 25

Belloche, A., Hennebelle, P., \& André, P. 2006, A\&A, 453, 145

Bertrang, G., Wolf, S., \& Das, H. S. 2014, A\&A, 565, A94

Bethell, T. J., Chepurnov, A., Lazarian, A., \& Kim, J. 2007, ApJ, 663, 1055

Bock, D. C.-J., Bolatto, A. D., Hawkins, D. W., et al. 2006, Proc. SPIE, 6267, 626713

Bodenheimer, P. 1995, ARA\&A, 33, 199

Bontemps, S., Andre, P., Terebey, S., \& Cabrit, S. 1996, A\&A, 311, 858

Brauer, R., Wolf, S., \& Reissl, S. 2016, A\&A, 588, A129

Caselli, P., Benson, P. J., Myers, P. C., \& Tafalla, M. 2002, ApJ, 572, 238

Chacón-Tanarro, A., Caselli, P., Bizzocchi, L., et al. 2017, A\&A, 606, A142

Chandler, C. J., \& Richer, J. S. 2000, ApJ, 530, 851

Chandler, C. J., Brogan, C. L., Shirley, Y. L., \& Loinard, L. 2005, ApJ, 632, 371

Chapman, N. L., Davidson, J. A., Goldsmith, P. F., et al. 2013, ApJ, 770, 151

Chen, X., Launhardt, R., \& Henning, T. 2007, ApJ, 669, 1058

Chen, X., Launhardt, R., \& Henning, T. 2009, ApJ, 691, 1729

Chen, X., Arce, H. G., Zhang, Q., et al. 2013, ApJ, 768, 110

Chiang, H.-F., Looney, L. W., \& Tobin, J. J. 2012, ApJ, 756, 168

Ching, T.-C., Lai, S.-P., Zhang, Q., et al. 2016, ApJ, 819, 159

Choi, M. 2001, ApJ, 553, 219

Choi, M. 2005, ApJ, 630, 976

Ciardi, A., \& Hennebelle, P. 2010, MNRAS, 409, L39

Ciardi, D. R., Telesco, C. M., Williams, J. P., et al. 2003, ApJ, 585, 392

Correia, J. C., Griffin, M., \& Saraceno, P. 2004, A\&A, 418, 607

Crimier, N., Ceccarelli, C., Maret, S., et al. 2010, A\&A, 519, A65

Crutcher, R. M. 2012, ARA\&A, 50, 29

Crutcher, R. M., Nutter, D. J., Ward-Thompson, D., \& Kirk, J. M. 2004, ApJ, 600, 279

Curiel, S., Raymond, J. C., Moran, J. M., Rodriguez, L. F., \& Canto, J. 1990, ApJ, 365, L85

Curiel, S., Torrelles, J. M., Rodríguez, L. F., Gómez, J. F., \& Anglada, G. 1999, ApJ, 527, 310

Curran, R. L., \& Chrysostomou, A. 2007, MNRAS, 382, 699

Davidson, J. A., Novak, G., Matthews, T. G., et al. 2011, ApJ, 732, 97

Di Francesco, J., Myers, P. C., Wilner, D. J., Ohashi, N., \& Mardones, D. 2001, ApJ, 562, 770

Di Francesco, J., Johnstone, D., Kirk, H., MacKenzie, T., \& Ledwosinska, E. 2008, ApJS, 175, 277

Dotson, J. L. 1996, ApJ, 470, 566

Enoch, M. L., Corder, S., Duchêne, G., et al. 2011, ApJS, 195, 21

Evans, II, N. J., Dunham, M. M., Jørgensen, J. K., et al. 2009, ApJS, 181, 321

Evans, II, N. J., Di Francesco, J., Lee, J.-E., et al. 2015, ApJ, 814, 22

Falceta-Gonçalves, D., Lazarian, A., \& Kowal, G. 2008, ApJ, 679, 537

Frau, P., Galli, D., \& Girart, J. M. 2011, A\&A, 535, A44

Gâlfalk, M., \& Olofsson, G. 2007, A\&A, 475, 281

Galli, D., Lizano, S., Shu, F. H., \& Allen, A. 2006, ApJ, 647, 374

Girart, J. M., Crutcher, R. M., \& Rao, R. 1999, ApJ, 525, L109

Girart, J. M., Rao, R., \& Marrone, D. P. 2006, Science, 313, 812

Girart, J. M., Frau, P., Zhang, Q., et al. 2013, ApJ, 772, 69

Girart, J. M., Estalella, R., Palau, A., Torrelles, J. M., \& Rao, R. 2014, ApJ, 780, L11

Gonçalves, J., Galli, D., \& Girart, J. M. 2008, A\&A, 490, L39

Goodman, A. A., Benson, P. J., Fuller, G. A., \& Myers, P. C. 1993, ApJ, 406, 528

Gueth, F., Guilloteau, S., \& Bachiller, R. 1996, A\&A, 307, 891

Harvey, D. W. A., Wilner, D. J., Myers, P. C., \& Tafalla, M. 2003, ApJ, 596, 383

Hennebelle, P., \& Ciardi, A. 2009, A\&A, 506, L29

Henning, T., Wolf, S., Launhardt, R., \& Waters, R. 2001, ApJ, 561, 871

Hildebrand, R. H. 1983, QJRAS, 24, 267

Hirano, N., Kameya, O., Nakayama, M., \& Takakubo, K. 1988, ApJ, 327, L69

Hirano, N., Ho, P. P. T., Liu, S.-Y., et al. 2010, ApJ, 717, 58

Hirota, T., Bushimata, T., Choi, Y. K., et al. 2008, PASJ, 60, 37

Hirota, T., Honma, M., Imai, H., et al. 2011, PASJ, 63, 1

Ho, P. T. P., Moran, J. M., \& Lo, K. Y. 2004, ApJ, 616, L1

Hoang, T., \& Lazarian, A. 2009, ApJ, 695, 1457

Holland, W. S., Robson, E. I., Gear, W. K., et al. 1999, MNRAS, 303, 659

Hull, C. L. H., Plambeck, R. L., Bolatto, A. D., et al. 2013, ApJ, 768, 159

Hull, C. L. H., Plambeck, R. L., Kwon, W., et al. 2014, ApJS, 213, 13

Hull, C. L. H., Girart, J. M., Tychoniec, Ł., et al. 2017, ApJ, 847, 92

Jennings, R. E., Cameron, D. H. M., Cudlip, W., \& Hirst, C. J. 1987, MNRAS, 226,461

Jones, T. J., Bagley, M., Krejny, M., Andersson, B.-G., \& Bastien, P. 2015, AJ, 149,31

Jones, T. J., Gordon, M., Shenoy, D., et al. 2016, AJ, 151, 156

Joos, M., Hennebelle, P., \& Ciardi, A. 2012, A\&A, 543, A128 
Jørgensen, J. K., Harvey, P. M., Evans, II, N. J., et al. 2006, ApJ, 645, 1246 Jørgensen, J. K., Bourke, T. L., Myers, P. C., et al. 2007, ApJ, 659, 479

Jørgensen, J. K., van der Wiel, M. H. D., Coutens, A., et al. 2016, A\&A, 595, A 117

Kataoka, A., Machida, M. N., \& Tomisaka, K. 2012, ApJ, 761, 40

Kataoka, A., Muto, T., Momose, M., et al. 2015, ApJ, 809, 78

Kauffmann, J., Bertoldi, F., Bourke, T. L., Evans, II, N. J., \& Lee, C. W. 2008 , A\&A, 487, 993

Keene, J., Davidson, J. A., Harper, D. A., et al. 1983, ApJ, 274, L43

Knude, J., \& Hog, E. 1998, A\&A, 338, 897

Koumpia, E., van der Tak, F. F. S., Kwon, W., et al. 2016, A\&A, 595, A51

Krumholz, M. R., Crutcher, R. M., \& Hull, C. L. H. 2013, ApJ, 767, L11

Kwon, W., Looney, L. W., Crutcher, R. M., \& Kirk, J. M. 2006, ApJ, 653, 1358

Kwon, W., Looney, L. W., Mundy, L. G., Chiang, H.-F., \& Kemball, A. J. 2009, ApJ, 696, 841

Kwon, W., Fernández-López, M., Stephens, I. W., \& Looney, L. W. 2015, ApJ, 814,43

Lai, S.-P., Crutcher, R. M., Girart, J. M., \& Rao, R. 2002, ApJ, 566, 925

Launhardt, R. 2001, in IAU Symp., The Formation of Binary Stars, eds. H. Zinnecker, \& R. Mathieu, 200, 117

Launhardt, R. 2004, in IAU Symp., Star Formation at High Angular Resolution, eds. M. G. Burton, R. Jayawardhana, \& T. L. Bourke, 221, 213

Launhardt, R., Stutz, A. M., Schmiedeke, A., et al. 2013, A\&A, 551, A98

Lazarian, A. 2007, J. Quant. Spectr. Rad. Transf., 106, 225

Lee, K. I., Dunham, M. M., Myers, P. C., et al. 2015, ApJ, 814, 114

Lee, J. W. Y., Hull, C. L. H., \& Offner, S. S. R. 2017, ApJ, 834, 201

Lefloch, B., Castets, A., Cernicharo, J., Langer, W. D., \& Zylka, R. 1998, A\&A, 334, 269

Li, Z.-Y., Krasnopolsky, R., \& Shang, H. 2013, ApJ, 774, 82

Li, H.-B., Goodman, A., Sridharan, T. K., et al. 2014, Protostars and Planets VI, 101

Liu, H. B., Lai, S.-P., Hasegawa, Y., et al. 2016, ApJ, 821, 41

Loinard, L., Zapata, L. A., Rodríguez, L. F., et al. 2013, MNRAS, 430, L10

Looney, L. W., Mundy, L. G., \& Welch, W. J. 2000, ApJ, 529, 477

Looney, L. W., Mundy, L. G., \& Welch, W. J. 2003, ApJ, 592, 255

Looney, L. W., Tobin, J. J., \& Kwon, W. 2007, ApJ, 670, L131

López-Sepulcre, A., Sakai, N., Neri, R., et al. 2017, A\&A, 606, A121

Machida, M. N., Matsumoto, T., Tomisaka, K., \& Hanawa, T. 2005, MNRAS, 362, 369

Machida, M. N., Inutsuka, S.-i., \& Matsumoto, T. 2007, ApJ, 670, 1198

Marrone, D. P. 2006, PhD thesis, Harvard University

Marrone, D. P., \& Rao, R. 2008, in Proc. SPIE, Millimeter and Submillimeter Detectors and Instrumentation for Astronomy IV, 7020, 70202B

Marvel, K. B., Wilking, B. A., Claussen, M. J., \& Wootten, A. 2008, ApJ, 685 285

Massi, F., Codella, C., Brand, J., di Fabrizio, L., \& Wouterloot, J. G. A. 2008 A\&A, 490, 1079

Matthews, B. C., \& Wilson, C. D. 2000, ApJ, 531, 868

Matthews, B. C., McPhee, C. A., Fissel, L. M., \& Curran, R. L. 2009, ApJS, 182, 143

Maury, A. J., André, P., Hennebelle, P., et al. 2010, A\&A, 512, A40

Maury, A. J., André, P., Men'shchikov, A., Könyves, V., \& Bontemps, S. 2011 A\&A, 535, A77

Maury, A. J., Belloche, A., André, P., et al. 2014, A\&A, 563, L2

Maury, A. J., Girart, J. M., Zhang, Q., et al. 2018, MNRAS, 477, 2760

Motte, F., \& André, P. 2001, A\&A, 365, 440

Ohashi, N., Saigo, K., Aso, Y., et al. 2014, ApJ, 796, 131

O’Linger, J., Wolf-Chase, G., Barsony, M., \& Ward-Thompson, D. 1999, ApJ, 515,696

Ossenkopf, V., \& Henning, T. 1994, A\&A, 291, 943

Oya, Y., Sakai, N., López-Sepulcre, A., et al. 2016, ApJ, 824, 88
Padoan, P., Goodman, A., Draine, B. T., et al. 2001, ApJ, 559, 1005 Palau, A., Zapata, L. A., Rodríguez, L. F., et al. 2014, MNRAS, 444, 833 Pech, G., Zapata, L. A., Loinard, L., \& Rodríguez, L. F. 2012, ApJ, 751, 78 Pelkonen, V.-M., Juvela, M., \& Padoan, P. 2009, A\&A, 502, 833 Pineda, J. E., Maury, A. J., Fuller, G. A., et al. 2012, A\&A, 544, L7 Planck Collaboration Int. XXXV. 2016, A\&A, 586, A138 Plunkett, A. L., Arce, H. G., Corder, S. A., et al. 2013, ApJ, 774, 22

Podio, L., Codella, C., Gueth, F., et al. 2016, A\&A, 593, L4

Poidevin, F., Bastien, P., \& Matthews, B. C. 2010, ApJ, 716, 893

Pudritz, R. E., \& Norman, C. A. 1983, ApJ, 274, 677

Qiu, K., Zhang, Q., Menten, K. M., Liu, H. B., \& Tang, Y.-W. 2013, ApJ, 779, 182

Rao, R., Crutcher, R. M., Plambeck, R. L., \& Wright, M. C. H. 1998, ApJ, 502, L75

Rao, R., Girart, J. M., Marrone, D. P., Lai, S.-P., \& Schnee, S. 2009, ApJ, 707, 921

Reipurth, B., Heathcote, S., \& Vrba, F. 1992, A\&A, 256, 225

Reipurth, B., Rodríguez, L. F., Anglada, G., \& Bally, J. 2002, AJ, 124, 1045

Rodríguez, L. F., Anglada, G., \& Curiel, S. 1997, ApJ, 480, L125

Sadavoy, S. I., Di Francesco, J., Andre, P., et al. 2014, ApJ, 787, L18

Saito, M., Sunada, K., Kawabe, R., Kitamura, Y., \& Hirano, N. 1999, ApJ, 518, 334

Santangelo, G., Codella, C., Cabrit, S., et al. 2015, A\&A, 584, A126

Schuller, F., Menten, K. M., Contreras, Y., et al. 2009, A\&A, 504, 415

Segura-Cox, D. M., Looney, L. W., Stephens, I. W., et al. 2015, ApJ, 798, L2

Segura-Cox, D. M., Harris, R. J., Tobin, J. J., et al. 2016, ApJ, 817, L14

Seifried, D., Banerjee, R., Pudritz, R. E., \& Klessen, R. S. 2015, MNRAS, 446, 2776

Shu, F. H., Najita, J. R., Shang, H., \& Li, Z.-Y. 2000, Protostars and Planets IV, 789

Stephens, I. W., Looney, L. W., Kwon, W., et al. 2013, ApJ, 769, L15

Straizys, V., Cernis, K., Kazlauskas, A., \& Meistas, E. 1992, Balt. Astron., 1, 149

Stutz, A. M., Rubin, M., Werner, M. W., et al. 2008, ApJ, 687, 389

Tafalla, M., \& Bachiller, R. 1995, ApJ, 443, L37

Tafalla, M., Kumar, M. S. N., \& Bachiller, R. 2006, A\&A, 456, 179

Tang, Y.-W., Ho, P. T. P., Koch, P. M., Guilloteau, S., \& Dutrey, A. 2013, ApJ, 763,135

Terebey, S., Chandler, C. J., \& Andre, P. 1993, ApJ, 414, 759

Terebey, S., \& Padgett, D. L. 1997, in IAU Symp., Herbig-Haro Flows and the Birth of Stars, eds. B. Reipurth, \& C. Bertout, 182, 507

Tobin, J. J., Looney, L. W., Mundy, L. G., Kwon, W., \& Hamidouche, M. 2007, ApJ, 659, 1404

Tobin, J. J., Hartmann, L., Chiang, H.-F., et al. 2011, ApJ, 740, 45

Tobin, J. J., Chandler, C. J., Wilner, D. J., et al. 2013, ApJ, 779, 93

Tobin, J. J., Looney, L. W., Wilner, D. J., et al. 2015, ApJ, 805, 125

Tobin, J. J., Looney, L. W., Li, Z.-Y., et al. 2016, ApJ, 818, 73

Watson, D. M., Bohac, C. J., Hull, C., et al. 2007, Nature, 448, 1026

Welch, W. J., Thornton, D. D., Plambeck, R. L., et al. 1996, PASP, 108, 93

Whittet, D. C. B., Hough, J. H., Lazarian, A., \& Hoang, T. 2008, ApJ, 674, 304

Wolf, S., Launhardt, R., \& Henning, T. 2003, ApJ, 592, 233

Wolf-Chase, G. A., Barsony, M., \& O’Linger, J. 2000, aj, 120, 1467

Wu, J., Dunham, M. M., Evans, II, N. J., Bourke, T. L., \& Young, C. H. 2007, AJ, 133, 1560

Yeh, S. C. C., Hirano, N., Bourke, T. L., et al. 2008, ApJ, 675, 454

Yen, H.-W., Takakuwa, S., \& Ohashi, N. 2010, ApJ, 710, 1786

Yen, H.-W., Takakuwa, S., \& Ohashi, N. 2011, ApJ, 742, 57

Yen, H.-W., Takakuwa, S., Ohashi, N., \& Ho, P. T. P. 2013, ApJ, 772, 22

Yen, H.-W., Koch, P. M., Takakuwa, S., et al. 2015, ApJ, 799, 193

Zhang, Q., Ho, P. T. P., Wright, M. C. H., \& Wilner, D. J. 1995, ApJ, 451, L71

Zhang, Q., Qiu, K., Girart, J. M., et al. 2014, ApJ, 792, 116 


\section{Appendix A: Source description}

In this section, we provide a quick description of the objects and of the $0.87 \mathrm{~mm}$ continuum observed with the SMA.

\section{B335}

B335 is an isolated Bok globule hosting a Class 0 protostar. Its luminosity is $\sim 1 L_{M_{\odot}}$ (Keene et al. 1983) and its mass $<2 M_{M_{\odot}}$. Ouflows. It possesses an east-west elongated, conical-shaped molecular outflow (PA: $80^{\circ}$; Hirano et al. 1988). Collimated ${ }^{12} \mathrm{CO}(2-1)$ jets (Yen et al. 2010) and Herbig-Haro (HH) objects (HH 119 A-F; Reipurth et al. 1992; Gâlfalk \& Olofsson 2007) are detected along this outflow axis.

Velocity field. $\mathrm{C}^{18} \mathrm{O}(2-1)$ and $\mathrm{H}^{13} \mathrm{CO}^{+}(1-0)$ interferometric observations have traced the rotational infalling motion of the envelope from radii of $\sim 20000$ down to $\sim 1000$ au. No clear rotational motion is detected on 100-500 au scales (Saito et al. 1999; Harvey et al. 2003; Yen et al. 2011) and the central circumstellar disk radius is estimated to be smaller than $100 \mathrm{au}$.

Our SMA observations. The SMA $0.87 \mathrm{~mm}$ continuum emission has a north-south elongated structure coherent with previous 1.3 mm observations of the object by Motte \& André (2001). The two protuberances observed in the north and southwest regions trace the edge of the cavity produced by the horizontal outflow (Yen et al. 2010).

\section{SVS13}

SVS13 is a multiple system in the NGC 1333 star-forming region. It possesses three main submillimeter continuum sources aligned in the northeast-southwest direction named $\mathrm{A}, \mathrm{B}$, and C (Looney et al. 2003). SVS13-A is a Class I protostar while SVS13-B and C are Class 0. A large filamentary structure encompasses the three sources at $450 \mu \mathrm{m}$ (Chandler \& Richer 2000) and at millimeter wavelengths (Hull et al. 2014). The combined luminosity for SVS13-A, B, and C is $45 L_{M_{\odot}}$ (Jennings et al. 1987). Emission at $70 \mu \mathrm{mis}$ detected toward SVS13-A and C but not toward SVS13-B, suggesting that the source is deeply embedded (Chen et al. 2009). A radio source called VLA3 has also been detected southwest of SVS13-A (Rodríguez et al. 1997).

Ouflows. SVS13-A is likely powering the strong northwestsoutheast HH7-11 outflow studied by Bachiller et al. (2000) and Plunkett et al. (2013). SVS13-B is known to drive a highly collimated $\mathrm{SiO}$ jet (Bachiller et al. 1998).

Velocity field. A symmetric velocity gradient of $28 \mathrm{~km} \mathrm{~s}^{-1} \mathrm{pc}^{-1}$ across SVS13-B and SVS13A/VLA3 has been detected using $\mathrm{N}_{2} \mathrm{H}^{+}$observations. This large gradient could suggest that the wide binary system is physically bound (Chen et al. 2009).

Our SMA observations. The SMA $0.87 \mathrm{~mm}$ observations trace the extended envelope around SVS13-A and SVS13-B. We resolve the two sources while SVS13-C is only marginally detected in the southwest. The continuum emission of SVS13-A is slightly elongated in the southwest direction toward the position of VLA3. The $0.87 \mathrm{~mm}$ emission of SVS13-B extends in the north-northwest direction. That extension aligns with that of the $\mathrm{SiO}$ jet driven by the protostar. The continuum emission also extends in the southwest direction of SVS13-B. The extension is also observed in the elongated $\mathrm{N}_{2} \mathrm{H}^{+}$map but not in the PdBI 1.4 $\mathrm{mm}$ and $3 \mathrm{~mm}$ observations, which could be linked with filtering effects affecting the PdBI maps (Chen et al. 2009).

\section{IC348-SMM2 / HH797}

SMM2 is a low-mass class 0 belonging to the IC 348 starforming region. The SMA observations also reveal a companion source, SMM2E, at $\sim 2400$ au in the northeast direction
(Palau et al. 2014) whose spectral energy distribution is typical of Class 0 objects.

Ouflows. SMM2 probably drives the HH797 jet, which has a very extended and collimated northwest-southeast outflow (Chen et al. 2013). Velocity asymmetries along the length of the flow were interpreted as jet rotation (Pech et al. 2012). A compact low-velocity outflow also seems to be associated with SMM2E.

Velocity field. $\mathrm{C}^{18} \mathrm{O}$ observations of the companion source SMM2E suggest that the envelope is probably rotating with a north-south axis close to the outflow direction (Palau et al. 2014).

Our SMA observations. Our $0.87 \mathrm{~mm}$ continuum map is consistent with that previously obtained by Palau et al. (2014) with SMA as well as far-IR continuum emission observed with Herschel. Both SMM2 and SMM2E are detected at $0.87 \mathrm{~mm}$, but SMME2 is not resolved.

\section{L1448C}

The L1448C complex (also called L1448-mm) hosts several embedded Class 0 protostars (Tobin et al. 2007).

Ouflows. It possesses well-collimated outflows (Hirano et al. 2010). The northern CO lobe is probably interacting with the neighboring source L1448N (Bachiller et al. 1995).

Velocity field. VLA observations of $\mathrm{NH}_{3}$ reveal velocity gradients perpendicular to the outflow as well as along the outflow axis. This suggests the presence of a self-gravitating structure around L1448C, which has an envelope that is both rotating and contracting at comparable velocities (Curiel et al. 1999).

Our SMA observations. Our SMA observations show that L1448C is elongated in the northwest-southeast direction, which is consistent with the elongation observed at $1.3 \mathrm{~mm}$ (Barsony et al. 1998). The southeast elongation encompasses the companion source L1448-mm B (2000 au separation) observed with Spitzer (Tobin et al. 2007) (also called L1448C(S); Jørgensen et al. 2006). A distortion is also observed in the southwest direction, where a $0.80 \mathrm{~mm}$ continuum emission has been observed by Barsony et al. (1998). This distortion and the northern elongation could also be tracing the edge of the outflow cavities.

\section{L1448N}

L1448N (or L1448 IRS3) is a multiple system. L1448N-A is located 2100 au north to L1448N-B. B is the strongest source at millimeter wavelengths (Looney et al. 2000) while A dominates at mid-infrared (Ciardi et al. 2003) and centimeter wavelengths (Curiel et al. 1990). The system is also connected to L1448NW located 20" north. Barsony et al. (1998) suggested that star formation in $\mathrm{L} 1448 \mathrm{~N}$ could have been induced by the strong outflow driven by L1448C (see also Kwon et al. 2006).

Ouflows. Two bipolar outflows originate from the system. The outflow driven by L1448N-A is nearly perpendicular to the line of sight. Kwon et al. (2006) showed that the two outflows are probably interacting with each other.

Velocity field. The inner $5-10^{\prime \prime}$ of L1448N presents a solidbody rotation while the outer envelope shows a flatter profile (Terebey \& Padgett 1997). $\mathrm{C}^{18} \mathrm{O}$ observations also reveal a strong velocity gradient $\left(>100 \mathrm{~km} \mathrm{~s}^{-1} \mathrm{pc}^{-1}\right.$; Gaudel et al., in prep.; Yen et al. 2015) perpendicular to the outflow direction within $20^{\circ}$ (Lee et al. 2015).

Our SMA observations. L1448N-A and B are resolved by the SMA while L1448NW is marginally detected. Both sources are elongated in the northeast-southwest direction, which is consis- 
tent with previous $230 \mathrm{GHz}$ and $0.87 \mathrm{~mm}$ SMA observations by Lee et al. (2015).

\section{L1448-2A}

L1448-2A (or L1448 IRS 2) is a low luminosity (5.2 $L_{\odot}$ ) Class 0 protostar located toward the western edge of the L1448 complex that seems to be evolving in isolation from L1448N and C.

Ouflows. High-velocity CO maps show that L1448-2A drives an outflow in the northwest-southeast direction (O'Linger et al. 1999). Additional studies from Wolf-Chase et al. (2000) suggested that the source is actually driving two distinct outflows, which could be a signature of an unresolved binary system. Velocity field. Using $\mathrm{C}^{18} \mathrm{O}$ observations, Yen et al. (2015) found a velocity gradient nearly perpendicular to the outflow axis.

Our SMA observations. The northeast-southwest extension of the envelope is confirmed by our SMA observation. Contrary to the CARMA $1.3 \mathrm{~mm}$ dust continuum presented in Hull et al. (2014), we do not observe elongation in the northwest-southeast direction. As this elongation is not observed at other wavelengths, we conclude that the elongation observed at $1.3 \mathrm{~mm}$ might be due to contamination by the outflow. The horns detected in the east and west direction trace the edges of the large outflow cavity.

\section{IRAS03282}

IRAS03282 is a highly embedded Class 0 object lying at $1^{\circ}$ southeast of NGC 1333 (Jørgensen et al. 2006). IRAS 03282 possesses two sources separated by 1.5" (Launhardt 2004).

Outflows. The source drives an outflow in which various velocity components are observed, in particular a high-velocity $\left(\sim 60 \mathrm{~km} \mathrm{~s}^{-1}\right)$ jet with fast molecular clumps detected along the outflow axis surrounded by a less collimated and more standard velocity $\left(\sim 20 \mathrm{~km} \mathrm{~s}^{-1}\right)$ outflow (Bachiller et al. 1991).

Velocity field. $\mathrm{N}_{2} \mathrm{H}^{+}$and $\mathrm{NH}_{3}$ observations were used to trace the large-scale velocity gradient of the envelope that is mostly distributed along the outflow direction (Tobin et al. 2011).

Our SMA observations. The $\mathrm{N}_{2} \mathrm{H}^{+}$and $\mathrm{NH}_{3}$ maps present an elongation along the north-south direction. Our SMA $0.87 \mathrm{~mm}$ map presents a similar elongation. The southern elongation is also very similar to that observed at $350 \mu \mathrm{m}$ by Wu et al. (2007) with SHARC-II.

\section{NGC 1333 IRAS4A}

NGC 1333 IRAS4A is a Class 0 object located in the south of NGC 1333. It hosts two sources (IRAS4A1 and IRAS4A2) in a close $\left(1.8^{\prime \prime}\right)$ binary pair that was resolved with interferometers at submillimeter and millimeter wavelengths using the SMA or BIMA (Looney et al. 2000; Girart et al. 2006; Jørgensen et al. 2007) and more recently ALMA (López-Sepulcre et al. 2017): IRAS4A1 dominates the submillimeter and millimeter emission. Outflows. An arcmin-long outflow, seen in high-velocity $\mathrm{CO}(3-$ 2) emission, is associated with IRAS4A2 and bends from a north-south orientation on small scales (Santangelo et al. 2015) to a position angle of $45 \circ$ on large scales Koumpia et al. (2016). This could be due to an intrinsic change of the gas propagation direction or a signature of jet precession. The launching direction of the outflow driven by IRAS4A1 is also oriented close to north-south direction and faster (Santangelo et al. 2015). Part of that $\mathrm{SiO}$ outflow (position angle of $-10^{\circ}$ ) was also reported by Choi (2001).

Velocity field. Detections of inverse P-Cygni profiles in the lines of common molecular species such as ${ }^{13} \mathrm{CO}$ or $\mathrm{CS}$ are interpreted as signs of strong infall motions of the outer cloud (Di Francesco et al. 2001; Jørgensen et al. 2007). Belloche et al. (2006) detected a centroid velocity gradient of about $10 \mathrm{~km} \mathrm{~s}^{-1} \mathrm{pc}^{-1}$, with a position angle of $38^{\circ}$, while
Ching et al. (2016) found a velocity gradient along the axis linking NGC 1333 IRAS4A1 and IRAS4A2. Both Belloche et al. (2006) and Gaudel et al (in prep.) showed however that the interpretation of these velocity gradients in terms of rotation is not straightforward in IRAS4A owing to the strong infall motions previously mentioned.

Our SMA observations. As already shown in Girart et al. (2006), the SMA $0.87 \mathrm{~mm}$ map presents a very compact structure extended in the A1-A2 direction. The common envelope extends in the northeast and southwest direction, in the same direction $\left(\sim 30-50^{\circ}\right)$ as $\left.i\right)$ the velocity gradient found in Belloche et al. (2006), ii) the outflow takes further from the protostar, and iii) that the large-scale B orientation observed by SCUBA (Matthews et al. 2009).

\section{NGC 1333 IRAS4B}

NGC 1333 IRAS4B is also a Class 0 protostar and belongs to the same $\mathrm{N}_{2} \mathrm{H}^{+}$arcmin-scale filament as IRAS4A. Submillimeter and millimeter observations reveal a second source located $11^{\prime \prime}$ on the east (IRAS4B2) (Looney et al. 2000). IRAS4B2 is not detected at centimeter wavelengths, suggesting that it is at a different stage of its evolution (Reipurth et al. 2002) or just not associated at all with IRAS4B. There is still some debate on smaller scales on whether the disk of IRAS 4B is observed face- or edge-on (Watson et al. 2007; Marvel et al. 2008).

Outflows. The source is driving an outflow whose axis is nearly in north-south direction. Its short dynamical timescale suggests that this outflow is very young (Choi 2001).

Velocity field. IRAS4B seems to be collapsing (Di Francesco et al. 2001; Belloche et al. 2006) but does not show clear signs of rotational motions at 1000 au scales (Yen et al. 2013).

Our SMA observations. The SMA $0.87 \mathrm{~mm}$ map of IRAS4B presents a very compact structure that seems to be extended in the north-south direction, namely the direction of the outflow emanating from this source. We do not observe the same east-west extension as seen at $1.3 \mathrm{~mm}$ by Hull et al. (2014) and Yen et al. (2013).

\section{IRAS16293}

IRAS16293 is a Class 0 protostar system located in the $\rho$ Ophiuchi molecular cloud. It possesses a bolometric luminosity of $\sim 25 L_{M_{\odot}}$ and a massive envelope (Correia et al. 2004; Crimier et al. 2010) surrounding two cores separated by 6", called A (south) and B (north). Crimier et al. (2010) suggested that IRAS16293A/B is a "separate envelope system" (following the classification proposed by Looney et al. 2003). IRAS16293A is an edge-on system and IRAS16293-B is nearly face-on (Jørgensen et al. 2016). The first dominates the system luminosity. The SMA observations also have enabled the separation of IRAS16293-A into two components, Aa and Ab, aligned along a position angle of $45^{\circ}$ (Chandler et al. 2005). No small-scale multiplicity has been found for IRAS16293-B.

Outflows. The source shows significant outflow activity. While IRAS16293-A seems to be driving the main east-west CO outflow, the driving source of the northwest-southeast compact outflow (PA of $145^{\circ}$ ) traced with $\mathrm{SiO}$ observations is still unclear (Yeh et al. 2008; Rao et al. 2009). This much younger outflow is probably a more robust tracer of the rotation axis, as recent studies revealed at various scales an almost edge-on rotational pattern whose axis match the $\mathrm{SiO}$ outflow orientation (Pineda et al. 2012; Girart et al. 2014). A one-sided bubble-like outflow structure originates from IRAS16293-B in the southeast direction (Loinard et al. 2013). 
Velocity field. In IRAS16293-A, $\mathrm{C}^{34} \mathrm{~S}$ observations reveal a clear velocity gradient along $\mathrm{PA} 40-45^{\circ}$, i.e., perpendicular to the $\mathrm{SiO}-$ traced outflow (Girart et al. 2014) probably because of the infalling motion of the rotating envelope. A disk-like rotation is also detected at 40-60 au with ALMA with the same orientation (Oya et al. 2016). In IRAS16293-B, recent ALMA observations reveal inverse P-Cygni profiles toward the center of IRAS16293-B in the $\mathrm{CH}_{3} \mathrm{OCHO}-\mathrm{A} \mathrm{CH} \mathrm{CH}_{3} \mathrm{OCHO}-\mathrm{E}$ and $\mathrm{H}_{2} \mathrm{CCO}$ lines, here again indicative of infall motions (Pineda et al. 2012). The source is observed face-on: no rotation has been detected. Our SMA observations. As shown in Rao et al. (2009), the SMA $0.87 \mathrm{~mm}$ observations separate the two A and B components. IRAS16293-B is brighter than IRAS16293-A. The continuum emission of the envelope extends to the north of IRAS16293-A and is also extended $\left(\sim 10^{\prime \prime}\right)$ toward its southeast direction, i.e., in the direction of the redshifted part of the $\mathrm{SiO}$ outflow.

\section{L1157}

L1157 is a low-mass Class 0 protostar and seems to be a single system (Tobin et al. 2013).

Outflows. It possesses a spectacular bipolar outflow asymmetric on large scales. Its prominent $\mathrm{CO}$ cavities are likely created by the propagation of large bow shocks (also traced via the $\mathrm{NH}_{3}$ or SiO molecular lines; Tafalla \& Bachiller 1995; Zhang et al. 1995; Gueth et al. 1996). This could be the sign that a timevariable collimated jet is driving the outflow (Podio et al. 2016). Velocity field. L1157 has a flattened and filamentary envelope presenting a weak velocity gradient along the filament - thus perpendicular to the outflow. Broad linewidths are observed in the inner envelope (see Kwon et al. 2015).
Our SMA observations. Our $0.87 \mathrm{~mm}$ observations are consistent with those presented in Chen et al. (2013). Neither Chen et al. (2013) nor our observations detected the elongation structure detected at $1.3 \mathrm{~mm}$ with Stephens et al. (2013) in the northern (outflow) direction. By contrast, we detect a similar eastern extension and western bump. This horizontal elongation is also observed at $350 \mu \mathrm{m}$ by Wu et al. (2007).

\section{CB230}

CB 230 is an isolated globule hosting a dense core, itself hosting two deeply embedded young stellar objects (YSOs). These are separated by $10^{\prime \prime}$ and are aligned in the east-west direction (Launhardt 2001, 2004). The eastern object, IRS2, is not detected beyond $24 \mu \mathrm{m}$ (Massi et al. 2008; Launhardt et al. 2013). Outflows. The north-south CO outflows seem to be driven by the western protostar IRS1. More recent $7 \mathrm{~mm}$ high-resolution observations of IRS1 led to the detection of two continuum sources separated by $\sim 100 \mathrm{au}$, which have an unresolved primary source at the origin of the outflow and an extended companion source located in a direction perpendicular to the outflow direction (Tobin et al. 2013).

Velocity field. CB230 presents a velocity gradient $>10 \mathrm{~km} \mathrm{~s}^{-1} \mathrm{pc}^{-1}$ increasing from east to west along the axis connecting the two embedded YSOs (Chen et al. 2007; Tobin et al. 2011). The gradient is perpendicular to the outflow axis.

Our SMA observations. The $0.87 \mathrm{~mm}$ continuum emission of CB230 is elongated in a boxy-shaped structure in the north-south direction, an elongation also observed at $1.3 \mathrm{~mm}$ by Hull et al. (2014). The southwest elongation could again be linked with emission tracing the edges of the north-south outflow cavity. 


\section{Appendix B: Stokes $Q$ and $U$, polarization intensity, and fraction maps of the sample}
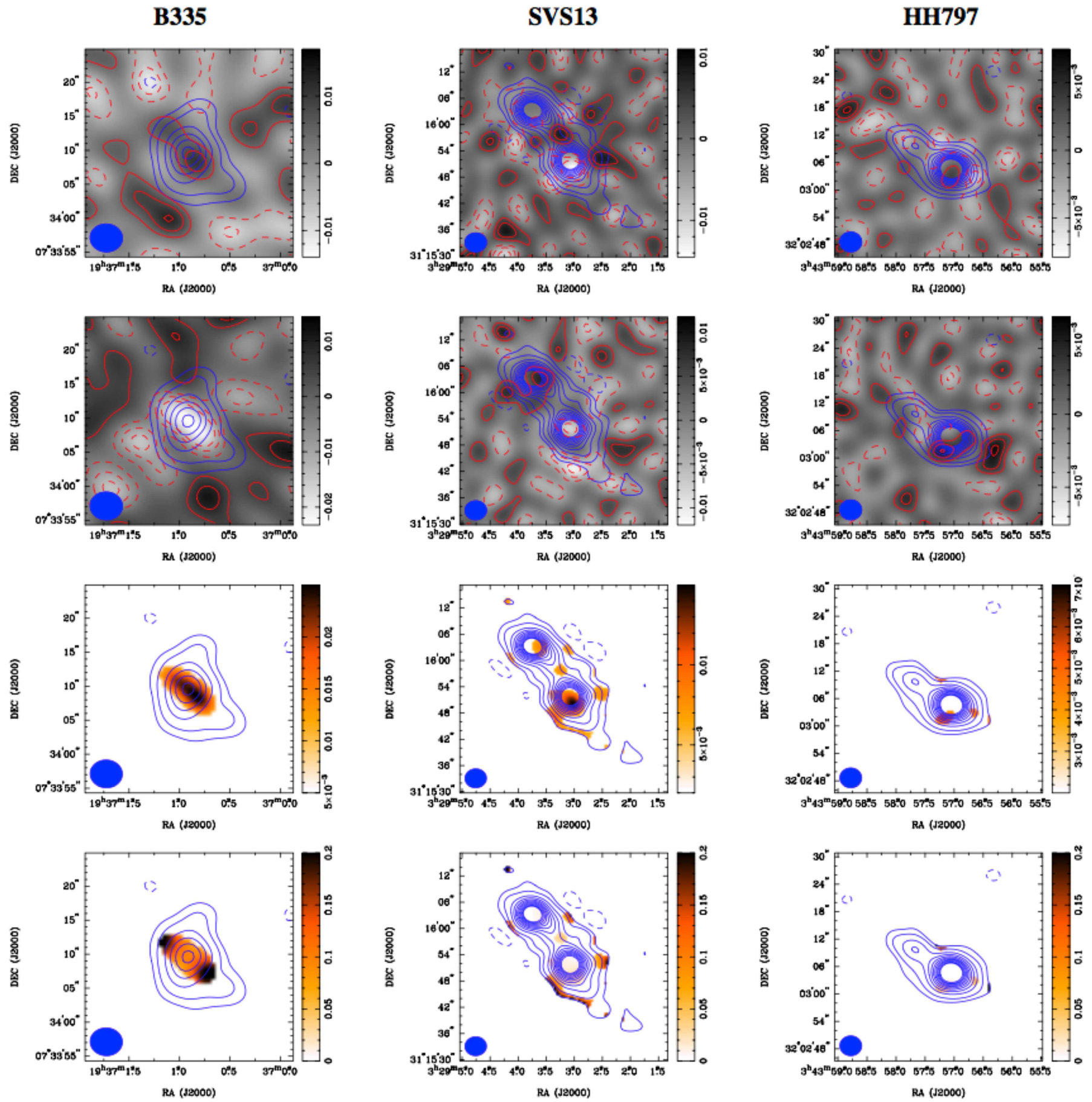

Fig. B.1. Polarization maps of, from left to right, B335, SVS13 and HH797. Two top panels: Stokes $Q$ and $U$ maps. Color scales are in mJy/beam. Stokes $I$ contours (same as in Fig. 2) appear in blue. Stokes $Q$ and $U$ contours appear in red and are $[-3,-2,-1,1,2,3] \sigma$. Third line: polarization intensity maps. The color scale is in mJy/beam. Fourth line: polarization fraction map. The filled ellipses on the bottom left corner indicate the synthesized beam of the SMA maps. Same for L1448C, N and 2A. Same for IRAS03282, L1157, and CB230. Same for NGC 1333 IRAS4A, NGC 1333 IRAS4B, and IRAS16293. 

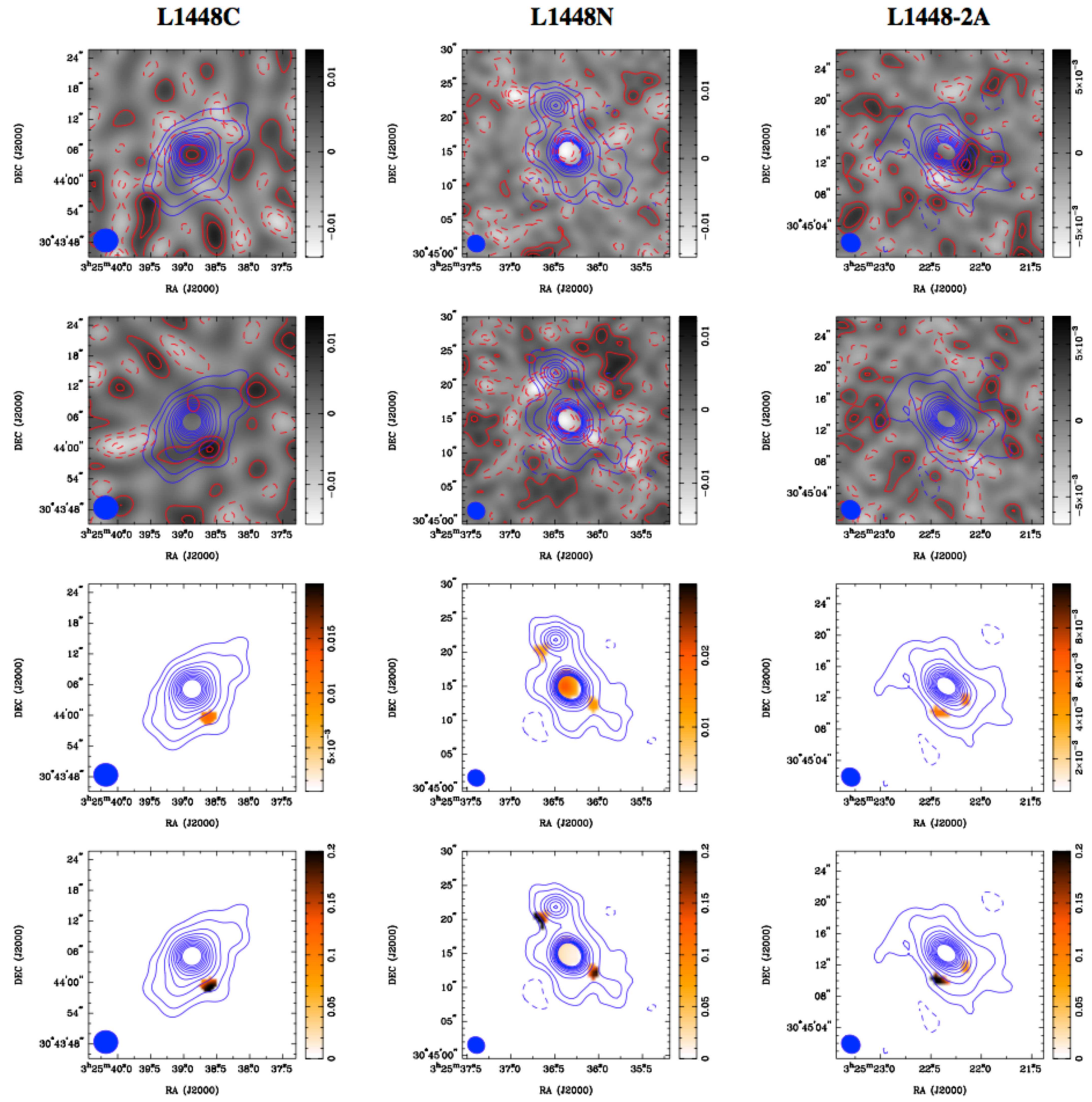

Fig. B.1. continued 
M. Galametz et al: Polarized dust emission in solar-type Class 0 protostars
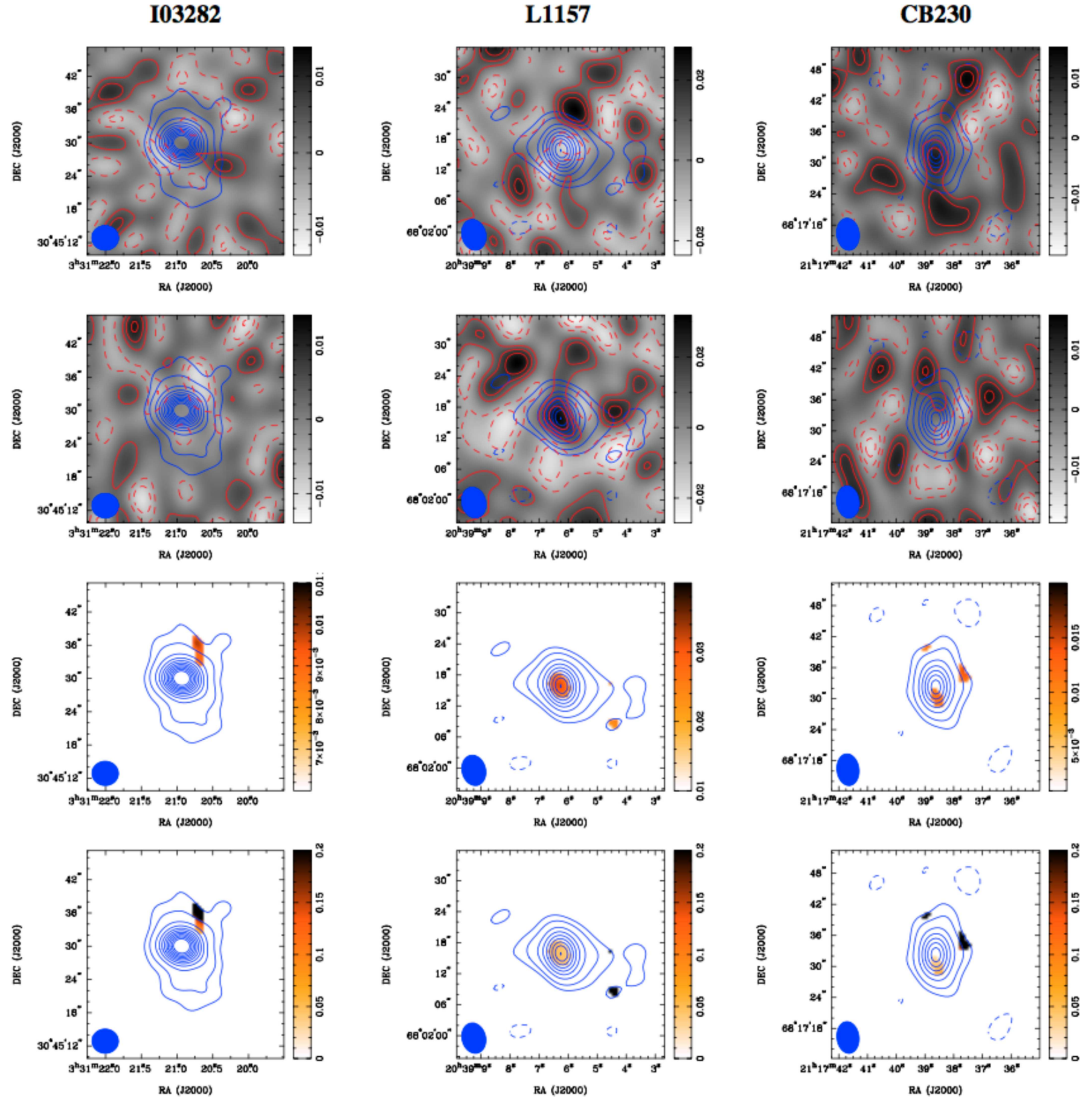

Fig. B.1. continued 

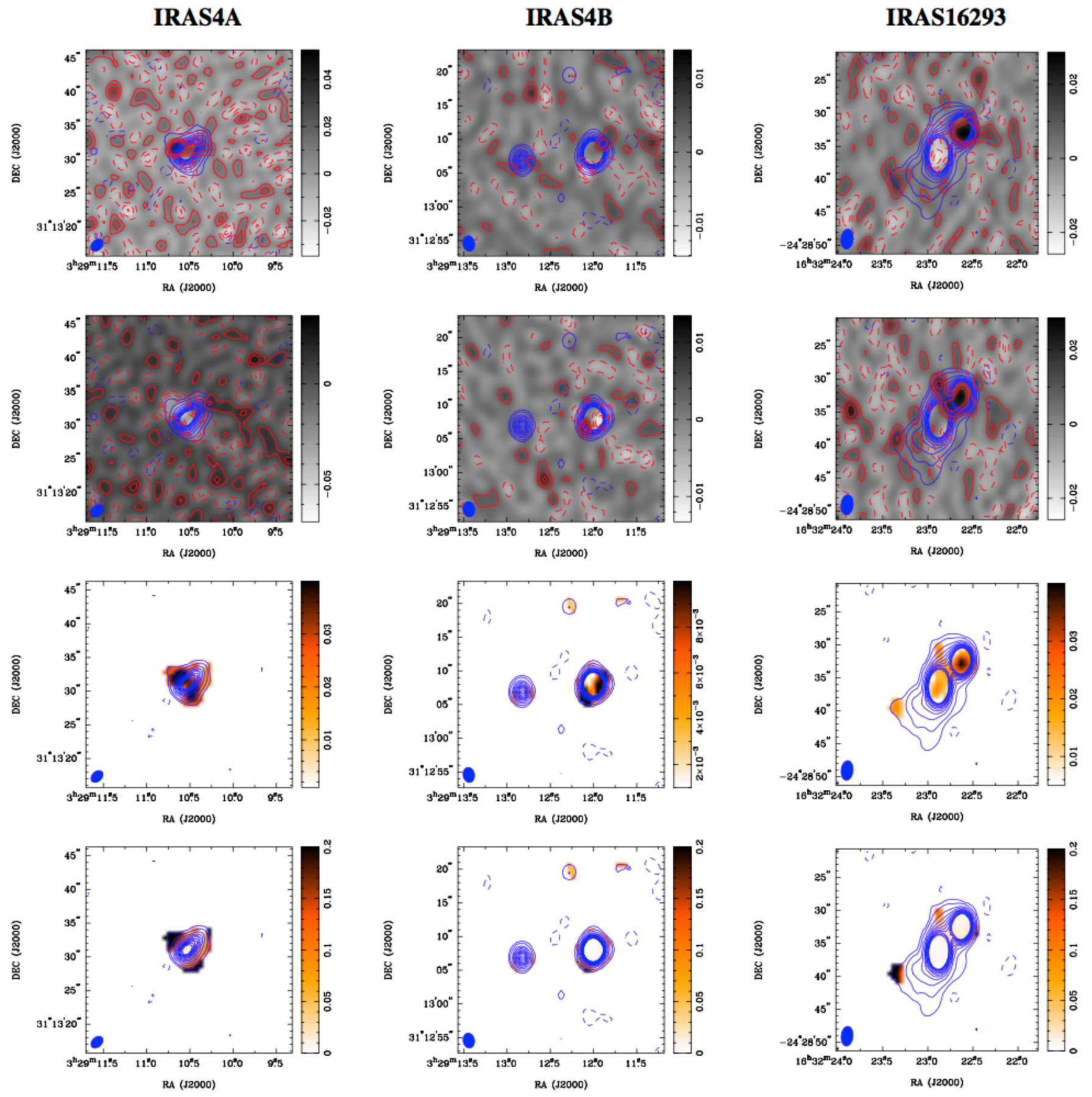

Fig. B.1. continued 